\title{
Efeitos do Halotano, Isoflurano e Sevoflurano sobre a Função Renal em Cães sob Pinçamento Aórtico Infra-Renal *
}

\section{Effects of Halothane, Isoflurane and Sevoflurane on Renal Function in Dogs under Infra-Renal Aortic Cross-Clamping}

\author{
Flora Margarida Barra Bisinotto, TSA ${ }^{1}$; José Reinaldo Cerqueira Braz, TSA ${ }^{2}$
}

\section{RESUMO}

Bisinotto FMB, Braz JRC - Efeitos do Halotano, Isoflurano e Sevoflurano sobre a Função Renal em Cães sob Pinçamento Aórtico Infra-Renal

JUSTIFICATIVA E OBJETIVOS: O pinçamento infra-renal da aorta abdominal pode produzir alterações renais. O objetivo do estudo foi avaliar os efeitos do halotano, isoflurano e sevoflurano sobre a função renal, em cães submetidos a pinçamento aórtico infra-renal.

MÉTODO: O estudo aleatório foi realizado em 30 cães, distribuídos em três grupos, de acordo com o anestésico halogenado utilizado durante a anestesia, em concentrações equipotentes de 0,75 CAM: $G H(n=10)$ - halotano a 0,67\%; GI $(n=10)$ - isoflurano a 0,96\%; e $G S(n=10)$ - sevoflurano a $1,8 \%$. Em todos os animais foi realizada ligadura infra-renal da aorta, por período de 30 minutos. Os atributos renais foram estudados nos momentos: C (controle), após 15 (Ao15) e 30 (Ao30) minutos de pinçamento aórtico, e após 15 (DAo15) e 30 (DAo30) minutos do despinçamento aórtico.

RESULTADOS: A depuração de água livre foi menor nos grupos GI e GS, em relação ao $\mathrm{GH}$, após o despinçamento aórtico $(p<0,05)$. Durante o pinçamento aórtico, nos três grupos, houve aumento do débito urinário, da excreção urinária de sódio e da depuração de sódio, e diminuição da osmolaridade urinária $(p<0,05)$. A resistência vascular renal e a fração de filtração aumentaram somente em GS $(p<0,05)$, enquanto a excreção fracionária de sódio aumentou em $\mathrm{GH}$ e GI $(p<0,05)$. Após o despinçamento aórtico, houve normalização dos atributos que haviam se alterado, com exceção da osmolaridade urinária, que continuou em níveis menores do que os do controle em todos os grupos $(p<0,05)$. A resistência vascular renal e a fração de filtração continuaram mais elevadas em GS, acompanhadas por diminuição do fluxo sangüíneo renal e da depuração de para-aminohipurato de sódio $(p<0,05)$.

CONCLUSÕES: No cão nas condições experimentais empregadas, a inalação de halotano e isoflurano a 0,75 CAM,

\footnotetext{
* Recebido do (Received from) Laboratório Experimental do CET/SBA do Departamento de Anestesiologia da Faculdade de Medicina de Botucatu (FMB), UNESP, no Programa de Pós-Graduação em Anestesiologia, Doutorado, com equipamentos adquiridos por meio de Auxílios à Pesquisa números 96/3302-0 e 97/09982 da FAPESP

1. Pós-Graduanda (Doutorado) do Programa de Pós-Graduação em Anestesiologia da FMB - UNESP. Bolsista do CNPq

2. Professor Titular do CET/SBA Departamento de Anestesiologia da FMB - UNESP

Apresentado (Submitted) em 28 de janeiro de 2003

Aceito (Accepted) para publicação em 01 de abril de 2003

Endereço para correspondência (Correspondence to)

Prof. Dr. José Reinaldo Cerqueira Braz

Dept ${ }^{\circ}$ de Anestesiologia da FMB - UNESP

Distrito de Rubião Junior

18618-970 - Botucatu, SP

(C) Sociedade Brasileira de Anestesiologia, 2003
}

mas não de sevoflurano, atenuou a principal alteração após o pinçamento infra-renal da aorta, que é o aumento da resistência vascular renal.

Unitermos: ANESTÉSICOS, Volátil: halotano, isoflurano, sevoflurano; ANIMAL: cão; CIRURGIA, Vascular: pinçamento aórtico infra-renal

\section{SUMMARY}

Bisinotto FMB, Braz JRC - Effects of Halothane, Isoflurane and Sevoflurane on Renal Function in Dogs under Infra-Renal Aortic Cross-Clamping

BACKGROUND AND OBJECTIVES: Infra-renal aortic cross-clamping is associated to renal effects. This study aimed at analyzing halothane, isoflurane and sevoflurane effects on renal function of dogs submitted to infra-renal aortic cross-clamping.

METHODS: This study involved 30 mixed-breed dogs randomly distributed in three groups, according to equipotent anesthetic doses (0.75 MAC) of inhaled anesthetics: $G H(n=10)$ $0.67 \%$ halothane; $\mathrm{GI}(n=10)-0.96 \%$ isoflurane; and GS ( $n=$ 10) - $1.8 \%$ sevoflurane. All animals were submitted to infra-renal aortic cross-clamping for 30 minutes. Renal parameters were evaluated at control (C), 15 (Ao15) and (Ao30) minutes after aortic cross-clamping, and 15 (DAo15) and 30 (DAo30) minutes after aortic unclamping.

RESULTS: Free water clearance was significantly lower in GI and $G S$ as compared to $\mathrm{GH}(p<0.05)$ after aortic unclamping. Urinary output, sodium urinary excretion and sodium clearance have significantly increased during aortic cross-clamping, while urinary osmolarity has decreased in all groups $(p<0.05)$. Renal vascular resistance and filtration fraction have increased during aortic cross-clamping in GS only, while sodium fractional excretion increased in $\mathrm{GH}$ and $\mathrm{Gl}(p<0.05)$. All renal parameters had returned to control levels after aortic unclamping, with the exception of urinary osmolality which has remained below control levels in all groups $(p<0.05)$. Renal vascular resistance and filtration fraction have remained higher in GS, followed by renal blood flow and PAH clearance decrease $(p<0.05)$.

CONCLUSIONS: In dogs under our experimental conditions, 0.75 MAC of halothane or isoflurane, but not 0.75 MAC of sevoflurane, have minimized renal vascular resistance increase, which is the major infra-renal aortic cross-clamping effect.

Key Words: ANESTHETICS, Volatile: halothane, isoflurane, sevoflurane, ANIMAL: dog; SURGERY, Vascular: infra-renal aortic cross-clamping

\section{INTRODUÇÃO}

s cirurgias para correção de aneurisma aórtico, mesmo Ino segmento infra-renal, podem estar associados à insuficiência renal, geralmente por necrose tubular aguda, ao redor de $5 \%$, que geralmente requer hemodiálise ${ }^{1}$ e apresenta ainda elevada taxa de mortalidade, ao redor de $50 \%$, 
apesar de todo avanço no diagnóstico e manuseio da insuficiência renal ${ }^{2}$. Quando a cirurgia é realizada no segmento aórtico supra-renal, a incidência de disfunção renal pode ser de $15 \%$ a $30 \%$ e quando requer a oclusão do segmento torácico a incidência pode chegar a $50 \%{ }^{3}$.

No pinçamento aórtico infra-renal podem ocorrer aumento da resistência vascular renal e diminuição do fluxo sangüíneo renal, ao redor de $30 \%$, que podem persistir após o despinçamento aórtico ${ }^{4}$ e mesmo alguns meses após a cirurgia ${ }^{5}$. No despinçamento aórtico infra-renal, normalmente ocorre redistribuição do fluxo sangüíneo nos rins, com diminuição do fluxo sangüíneo renal cortical e do ritmo de filtração glomerular ${ }^{6,7}$ que não parecem estar correlacionados às alterações de débito cardíaco ou da pressão arterial média ${ }^{7,8}$, mas sim ao aumento da resistência vascular renal ${ }^{9}$. A diminuição do volume urinário, que pode ocorrer, parece não ter correlação com a redução do ritmo de filtração glomerular ${ }^{7} \mathrm{e}$ com o grau de insuficiência renal no pós-operatório ${ }^{10}$.

Vários fatores têm contribuído para a redução da mortalidade durante a cirurgia para a correção de aneurisma abdominal, como melhoria da técnica cirúrgica, intervenção cirúrgica mais precoce, melhor seleção dos pacientes, avanços na monitorização, surgimento de novas drogas e técnicas anestésicas, e aumento dos cuidados intensivos no pós-operatório ${ }^{9}$.

Os anestésicos podem apresentar importante papel na patogênese das alterações cardíacas e renais durante o pinçamento aórtico, pelos seus efeitos sobre a hemodinâmica cardiovascular e a liberação de hormônios mediadores. Assim, o halotano parece estar associado a maiores alterações renais do que o isoflurano, quando utilizado em doses equipotentes em pacientes submetidos à cirurgia de reconstrução aórtica sob pinçamento aórtico infra-renal ${ }^{11,12}$, provavelmente por diminuir o débito cardíaco, fluxo sangüíneo renal e ritmo de filtração glomerular em maior proporção do que o isoflurano. Segundo os autores ${ }^{11,12}$, esses resultados sugerem que a anestesia com halotano está associada com vasoconstrição renal, que não ocorre com o isoflurano. Com relação ao sevoflurano, as primeiras pesquisas não encontraram alterações renais importantes, principalmente com a capacidade de concentração renal, tanto no homem ${ }^{13,14}$ como em animais de experimentação ${ }^{15,16}$. Mas a preocupação com a função de concentração renal é relevante, porque o sevoflurano em seu metabolismo forma íns flúor, que potencialmente podem provocar lesão tubular renal. Há preocupação também com a formação do fluormetil-2,2 -diflúor - 1 - (trifluormetil) vinil éter, também conhecido como composto $A$, porque o sevoflurano pode formar produtos metabólicos na presença de absorvedores de dióxido de carbono, pela ação da enzima $\beta$-liase renal. Esta substância é nefrotóxica em ratos ${ }^{17,18}$, mas não no homem, por causa da menor atividade da $\beta$-liase neste último. Também, o composto Aé formado em baixas concentrações nos circuitos anestésicos, mesmo nos fechados, utilizando-se baixo fluxo de gases frescos. Por isso parece ser desprovido de efeito nefrotóxico no homem ${ }^{19}$.
Mais recentemente, alguns autores verificaram que o sevoflurano, quando empregado em baixo fluxo de gases frescos, pode determinar alterações renais, caracterizadas por aumento da excreção urinária de albumina e glicose, e de algumas enzimas tubulares ${ }^{20,21}$. No entanto, esses resultados têm sido questionados por outros autores ${ }^{14,22,23}$ por acreditarem que os melhores marcadores de alterações renais ainda continuam a ser os níveis plasmáticos de uréia e creatinina e por não existirem evidências significativas para se creditarem às alterações enzimáticas tubulares uma indicação importante de ocorrência de lesão renal com o sevoflurano. Por outro lado, em pacientes com insuficiência renal estável e submetidos à cirurgia eletiva sob anestesia com sevoflurano ou isoflurano em baixo fluxo de gases frescos, os autores não observaram alteração da função renal ${ }^{23,24}$.

Frente aos resultados encontrados na literatura, o FDA não liberou o uso do sevoflurano na presença de fluxo de gases frescos menor ou igual a um litro.

Não há na literatura estudos das ações do sevoflurano no aparelho renal em situações cirúrgicas que possam ocasionar alterações renais, como as que podem ocorrer durante a cirurgia de reconstrução aórtica.

A presente pesquisa tem como objetivo o estudo comparativo dos efeitos do halotano, isoflurano e sevoflurano, em concentrações equipotentes, sobre a função renal de cães submetidos a pinçamento aórtico infra-renal, com a finalidade de verificar qual dos agentes inalatórios "protege" melhor os rins dos efeitos provocados pelo pinçamento e despinçamento aórticos.

\section{MÉTODO}

Após aprovação pela Comissão de Ética em Pesquisa Animal, foram utilizados 30 cães adultos, de ambos os sexos, pesando entre 18 a $20 \mathrm{~kg}$. Os cães foram distribuídos aleatoriamente em três grupos, com 10 animais em cada grupo. $O$ experimento constou de duas fases: na primeira, foi feita indução anestésica, intubação traqueal, instalação de ventilação artificial, manutenção anestésica com halotano, isoflurano ou sevoflurano, dependendo do grupo estudado, monitorização da ventilação, oxigenação, hemodinâmica cardiovascular, função renal e temperatura, seguida de laparotomia mediana para preparação da ligadura aórtica infra-renal; e na segunda fase, foi realizado o pinçamento aórtico por 30 minutos, seguido da retirada da ligadura aórtica. Os grupos se diferenciaram como segue:

GH - Manutenção anestésica com halotano a 0,67\% $(0,75$ da concentração alveolar mínima - CAM);

GI - Manutenção anestésica com isoflurano a 0,96\% (0,75 CAM);

GS - Manutenção anestésica com sevoflurano a 1,8\% (0,75 CAM).

A CAM utilizada dos halogenados no cão seguiu a proposição de Kazama e Ikeda (1988) ${ }^{25}$. 


\section{Seqüência Experimental}

Após jejum alimentar de 12 horas, mas com livre acesso à água, os animais, após indução anestésica com propofol $\left(5,5 \mathrm{mg} \cdot \mathrm{kg}^{-1}\right)$ e cloreto de alcurônio $\left(0,2 \mathrm{mg} \cdot \mathrm{kg}^{-1}\right)$, por via venosa, foram colocados em goteira de Claude Bernard em sala de experimentação cuja temperatura foi mantida por ar condicionado (quente/frio) entre 24 e $25^{\circ} \mathrm{C}$, realizando-se a seguir:

1. Intubação orotraqueal, utilizando-se tubo traqueal de diâmetro interno de 8,5 ou $9 \mathrm{~mm}$ provido de balonete de alta complacência, e instalação de ventilação controlada a volume, empregando-se o respirador Ohmeda (EUA) do aparelho de anestesia Excel mod. $210 \mathrm{SE}$ (Ohmeda - EUA) e fluxo de ar comprimido (1,2 L. $\left.\mathrm{min}^{-1}\right)$ e oxigênio $\left(0,8\right.$ L. $\left.\mathrm{min}^{-1}\right)$, em sistema circular valvular semifechado com reabsorvedor de gás carbônico (cal sodada). Empregou-se fração inspirada de oxigênio entre $40 \%$ e $50 \%$, volume corrente de $20 \mathrm{ml}^{\mathrm{kg}} \mathrm{kg}^{-1}$ e freqüência respiratória de 10 a 15 movimentos. in $^{-1}$ para manutenção da pressão expiratória final de $\mathrm{CO}_{2}$ entre 30 e 35 $\mathrm{mmHg}$;

2. Instalação do aparelho AS3 da Datex-Engstrom (Finlândia) para leitura e registro dos parâmetros ventilatórios, dos gases anestésicos, hemodinâmicos, de oxigenação e de temperatura;

3. A inalação do agente halogenado foi feita por meio de vaporizador calibrado específico para cada halogenado (Ohmeda, EUA). A concentração inspirada e expirada de cada agente halogenado, de $\mathrm{O}_{2}$ e de $\mathrm{CO}_{2}$ foi controlada pelo módulo de Analisador de Gases do aparelho AS3 da Datex-Engstrom (Finlândia), utilizando-se captador de amostra de gases junto à válvula em "Y" do circuito respiratório. Empregou-se inicialmente uma CAM do halogenado (halotano a $0,89 \%$, isoflurano a $1,3 \%$ e sevoflurano a $2,4 \%$ ) de acordo com a concentração expirada;

4. Instalação do eletrocardiógrafo de três canais (derivação $D_{\text {III }}$, sensor do termômetro no terço inferior do esôfago e sensor em forma de pinça colocado na língua do animal, para leitura da saturação periférica de oxigênio da oxihemoglobina;

5. Dissecção e cateterismo da veia femoral direita para administração de drogas, como doses complementares de cloreto de alcurônio $\left(0,06 \mathrm{mg} \cdot \mathrm{kg}^{-1}\right)$, e coleta de sangue venoso;

6. Dissecção e cateterismo da veia femoral esquerda para infusão contínua da solução de Ringer com lactato (18 $\mathrm{ml} . \mathrm{kg}^{-1}$.). Após 30 minutos, foi feito o "prime" das soluções de creatinina (3\%) e ácido para-aminohipúrico (PAH) $(0,4 \%)$, sendo utilizado $1 \mathrm{ml} . \mathrm{kg}^{-1}$ da solução. A seguir, colocou-se solução de Ringer $\left(18 \mathrm{ml} \cdot \mathrm{kg}^{-1} \cdot \mathrm{h}^{-1}\right)$ com PAH $(0,08 \%)$ e creatinina $(0,2 \mathrm{~g} \%)$, administrando-se por minuto, até o final do experimento, $0,6 \mathrm{mg} \cdot \mathrm{kg}^{-1} \mathrm{de}$ creatinina e $0,24 \mathrm{mg} \cdot \mathrm{kg}^{-1}$ de $\mathrm{PAH}$, por meio de bomba de infusão de dois canais Anne, da Abbott (EUA);
7. Dissecção e cateterismo da artéria femoral esquerda para medida da pressão aórtica e controle do pinçamento e despinçamento aórticos;

8. Dissecção e cateterismo da artéria axilar esquerda para medida da pressão arterial média e coleta de sangue para medida do $\mathrm{pH}$ e gases sangüíneos no aparelho Chiron Diagnostics, mod. Rapidlab 865 (Inglaterra);

9. Dissecção e cateterismo da veia jugular externa direita com introdutor $8,5 \mathrm{~F}$ e passagem de cateter de Swan-Ganz 7F até a artéria pulmonar, para medida das pressões e do débito cardíaco, por termodiluição;

10. Cateterismo vesical para coleta de urina em provetas graduadas;

11. Realização de laparotomia mediana e dissecção infra-renal da aorta. Colocação de fita cardíaca ao redor da aorta, imediatamente após a emergência das artérias renais, para posterior ligadura aórtica. A fita cardíaca foi transpassada em pequeno tubo plástico de $15 \mathrm{~cm}$. A incisão cirúrgica foi, em seguida, fechada ao redor do tubo plástico;

12. Após o preparo, reduziu-se a CAM dos halogenados, segundo o grupo estudado, de uma para 0,75 CAM, iniciando-se o período de estabilização hemodinâmica, com duração de 30 minutos;

13. Medida dos atributos e coleta de sangue (venoso e arterial) e de urina (momento controle);

14. Injeção de heparina, por via venosa, na dose de 70 Ul. $\mathrm{kg}^{-1} \mathrm{e}$, após 3 minutos, realização da ligadura infra-renal da aorta, avançando-se o tubo plástico ao longo da fita cardíaca até que não mais houvesse registro de pressão na artéria femoral;

15. Medida dos atributos e coleta de sangue e urina após 15 e 30 minutos da ligadura aórtica (momentos Ao15 e Ao30);

16. Retirada da ligadura aórtica, medida dos atributos e coleta de sangue e urina após 15 e 30 minutos da retirada da ligadura aórtica (momentos DAo15 e DAo30);

17. Término do experimento e sacrifício do animal com excesso de anestésico (pentobarbital sódico).

\section{Atributos Estudados}

Os atributos estudados foram: antropométricos - peso $(\mathrm{kg})$, comprimento $(\mathrm{cm})$ e superfície corporal $\left(\mathrm{m}^{2}\right)$; sexo; hemodinâmicos - freqüência cardíaca (FC), pressão arterial média (PAM), pressão média do átrio direito (PAD), pressão média da artéria pulmonar (PAP), pressão da artéria pulmonar ocluída (PAPO), índice cardíaco (IC), índice sistólico (IS) e índice de resistência vascular sistêmica (IRVS); função renal - depuração de para-aminohipurato de sódio (DPAH) para medida do fluxo plasmático renal (FPR), fluxo sangüíneo renal $(F S R=F P R / 1-H t)$, resistência vascular renal $(R V R=P A M x$ $80 / F S R \times 10^{-3}$ ), débito urinário (DU), osmolalidade urinária (UOsm), depuração de creatinina (DCr), para determinação do ritmo de filtração glomerular (RFG), fração de filtração (FF $=\mathrm{DC} / \mathrm{DPAH})$, depuração osmolar $($ Dosm $=$ Uosm $\mathrm{x}$ $\mathrm{DU} /$ Posm), depuração de água livre ( $\mathrm{DH}_{2} \mathrm{O}=\mathrm{DU}$ - Dosm), ex- 
creção urinária de sódio (EUNa = DU x UNa), depuração de sódio (DNa $\times$ DU/PNa) e excreção fracionária de sódio (DNa/DCr x 100); sangüíneos - hematócrito $(\mathrm{Ht})$, sódio plasmático ( $\mathrm{PNa})$, osmolalidade plasmática (Posm), $\mathrm{pH}$ arterial $(\mathrm{pHa})$, pressão parcial de gás carbônico arterial $\left(\mathrm{PaCO}_{2}\right) \mathrm{e}$ metabólicos - temperatura esofagiana ( $\left.T_{E s o f}\right)$.

\section{Análise Estatística}

A análise estatística dos resultados foi efetuada utilizando-se a Análise de Perfil ${ }^{26}$. Para as variáveis antropométricas, utilizou-se ANOVA e para a distribuição dos sexos, utilizou-se o teste Qui-quadrado $\left(\mathrm{X}^{2}\right)$. As estatísticas foram consideradas significantes quando $p<0,05$.

\section{RESULTADOS}

Não houve diferença significante entre os grupos em relação aos valores antropométricos e a distribuição do sexo (Tabela I).
Tabela I - Atributos Antropométricos e Distribuição do Sexo nos Grupos

\begin{tabular}{lcccc}
\hline Grupos & $\begin{array}{c}\text { Peso } \\
(\mathrm{kg})^{*}\end{array}$ & $\begin{array}{c}\text { Comprimento } \\
(\mathrm{cm})^{*}\end{array}$ & $\begin{array}{c}\text { Superfície Corporal } \\
\left(\mathrm{m}^{2}\right)^{*}\end{array}$ & $\begin{array}{c}\text { Sexo } \\
(\mathrm{M} / \mathrm{F})\end{array}$ \\
\hline $\mathrm{GH}$ & $18,3 \pm 1,6$ & $101,2 \pm 7,8$ & $0,702 \pm 0,067$ & $7 / 3$ \\
$\mathrm{GI}$ & $19,9 \pm 2,0$ & $103,0 \pm 5,6$ & $0,731 \pm 0,043$ & $6 / 4$ \\
$\mathrm{GS}$ & $19,7 \pm 2,1$ & $104,1 \pm 5,2$ & $0,736 \pm 0,061$ & $8 / 2$ \\
\hline
\end{tabular}

Não houve diferença significante entre os grupos $(p>0,05)$

* Valores expressos pela Média $\pm \mathrm{DP}$

A freqüência cardíaca foi o único atributo hemodinâmico que apresentou alteração significante entre os grupos, sendo os valores de $\mathrm{GH}$ menores do que os dos demais grupos durante o pinçamento aórtico (Tabela II). Durante o pinçamento aórtico, houve aumento da PAM, PAD e IS em todos os grupos, enquanto os valores da PAP aumentaram em GHe Gl e o IC aumentou em GI (Tabela II). Após o despinçamento aórtico, os valores dos atributos hemodinâmicos que haviam se elevado retornaram aos níveis do controle, com exceção dos

Tabela II - Valores da Freqüência Cardíaca (FC), Pressão Arterial Média (PAM), Pressão do Átrio Direito (PAD), Pressão da Artéria Pulmonar (PAP), Pressão da Artéria Pulmonar Ocluída (PAPO), Índice Cardíaco (IC), Índice Sistólico (IS) e Índice de Resistência Vascular Sistêmica (IRVS) nos Momentos dos Grupos Estudados (Média \pm DP)

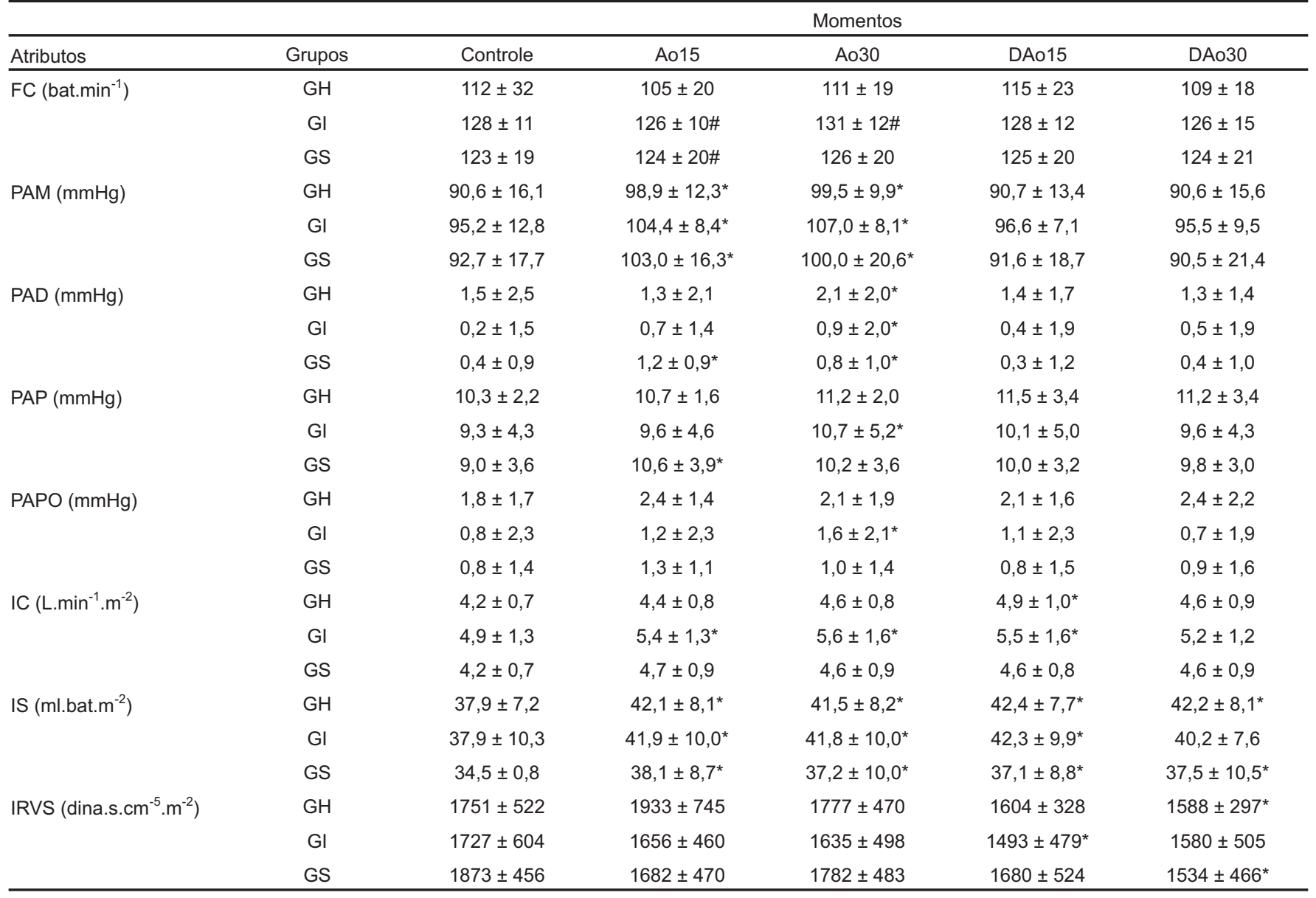

\# p < 0,05: diferença significante entre os grupos em relação ao $\mathrm{GH}$

* $p<0,05$ : diferença significante em relação ao momento controle do mesmo grupo 
valores do IC e IS, que continuaram elevados (Tabela II). O IRVS, que não se alterou significantemente durante o pinçamento aórtico em nenhum dos grupos, apresentou diminuição significante dos valores nos três grupos.

A depuração de água livre foi o único atributo renal que apresentou alteração significante entre os grupos, sendo os valores de GI e GS menores do que os de $\underline{\mathrm{GH}}$ após o despinçamento aórtico. Durante o pinçamento aórtico, os três grupos apresentaram aumento significante do DU, da EUNa e da DNa, e diminuição da Uosm (Tabela III). ARVR aumentou no grupo GS (Figura 1), acompanhada por aumento da FF (Tabela IV). A EFNa aumentou nos grupos GH e GI (Tabela III). Após o despinçamento aórtico, houve normalização dos atributos que haviam se elevado, com exceção da Uosm, que continuou em níveis mais baixos em relação aos do controle em todos os grupos (Tabela III), e da RVR (Figura 1) e FF (Tabela IV) em GS, que continuaram significantemente mais elevadas, acompanhadas por diminuição significante do FSR e da DPAH (Figura 2 e Tabela IV, respectivamente). A DCr não se alterou nos três grupos experimentais nos momentos estudados (Tabela IV).

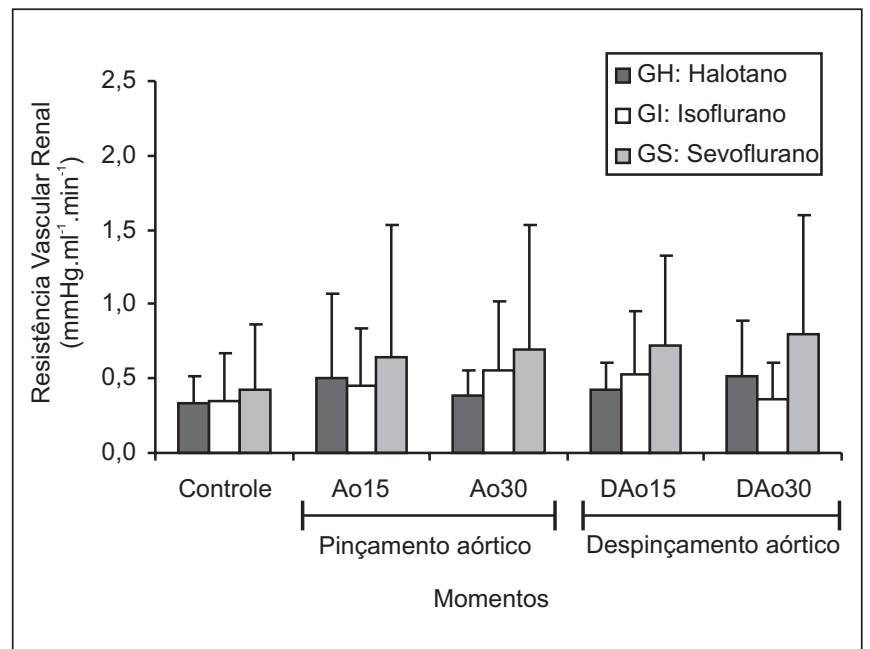

Figura 1 - Resistência Vascular Renal (RVR). Valores Obtidos nos Momentos dos Grupos Estudados com Indicação do Resultado Estatístico Obtido (Média \pm DP)

${ }^{*} p<0,05:$ GS: Controle < $($ Ao30 $=$ DAo15 = DAo30 $)$

Tabela III - Valores do Débito Urinário (DU), Osmolaridade Urinária (Uosm), Depuração Osmolar (Dosm), Depuração de Água Livre $\left(\mathrm{DH}_{2} \mathrm{O}\right)$, Excreção Urinária de Sódio (EUNa). Depuração de Sódio (DNa) e Excreção Fracionária de Sódio (EFNa) nos Momentos dos Grupos Estudados (Média \pm DP)

\begin{tabular}{|c|c|c|c|c|c|c|}
\hline \multirow[b]{2}{*}{ Atributos } & \multirow[b]{2}{*}{ Grupos } & \multicolumn{5}{|c|}{ Momentos } \\
\hline & & Controle & Ao15 & Ao30 & DAo15 & DAo30 \\
\hline \multirow[t]{3}{*}{$\mathrm{DU}\left(\mathrm{ml} \cdot \mathrm{min}^{-1} \cdot \mathrm{kg}^{-1}\right)$} & $\mathrm{GH}$ & $0,14 \pm 0,11$ & $0,18 \pm 0,09^{*}$ & $0,19 \pm 0,09^{*}$ & $0,14 \pm 0,08$ & $0,14 \pm 0,06$ \\
\hline & $\mathrm{GI}$ & $0,10 \pm 0,06$ & $0,14 \pm 0,07$ & $0,15 \pm 0,06^{*}$ & $0,09 \pm 0,04$ & $0,12 \pm 0,07$ \\
\hline & GS & $0,10 \pm 0,11$ & $0,16 \pm 0,13^{*}$ & $0,15 \pm 0,09^{*}$ & $0,13 \pm 0,08$ & $0,11 \pm 0,07$ \\
\hline \multirow[t]{3}{*}{ Uosm (mOsm.kg ${ }^{-1}$ de água) } & $\mathrm{GH}$ & $487 \pm 260$ & $378 \pm 157^{*}$ & $345 \pm 157^{*}$ & $370 \pm 182^{*}$ & $377 \pm 182^{*}$ \\
\hline & $\mathrm{GI}$ & $700 \pm 238$ & $559 \pm 245^{*}$ & $486 \pm 196^{*}$ & $498 \pm 210^{*}$ & $488 \pm 179^{*}$ \\
\hline & GS & $738 \pm 209$ & $564 \pm 172^{*}$ & $557 \pm 168^{*}$ & $577 \pm 147^{*}$ & $598 \pm 161^{*}$ \\
\hline \multirow[t]{3}{*}{$\operatorname{Dosm}\left(\mathrm{ml}_{\mathrm{min}}\right)$} & $\mathrm{GH}$ & $3,29 \pm 1,72$ & $4,24 \pm 1,80$ & $3,86 \pm 1,85$ & $3,00 \pm 1,17$ & $3,34 \pm 1,66$ \\
\hline & $\mathrm{GI}$ & $4,41 \pm 2,72$ & $4,88 \pm 2,20$ & $4,65 \pm 2,13$ & $2,91 \pm 1,59$ & $3,57 \pm 2,11$ \\
\hline & GS & $4,33 \pm 3,20$ & $5,25 \pm 3,18$ & $5,13 \pm 2,73$ & $4,40 \pm 1,63$ & $4,09 \pm 1,57$ \\
\hline \multirow[t]{2}{*}{$\mathrm{DH}_{2} \mathrm{O}\left(\mathrm{ml} \cdot \mathrm{min}^{-1}\right)$} & $\mathrm{GH}$ & $-0,85 \pm 1,18$ & $-0,89 \pm 2,26$ & $-0,45 \pm 1,81$ & $-0,39 \pm 1,21$ & $-0,74 \pm 1,07$ \\
\hline & GS & $-2,28 \pm 1,32$ & $-2,05 \pm 1,60$ & $-2,22 \pm 1,96$ & $-1,89 \pm 0,53 \#$ & $-1,87 \pm 0,86$ \\
\hline \multirow[t]{3}{*}{ EUNa $\left(\mu \mathrm{Eq} \cdot \mathrm{min}^{-1}\right)$} & $\mathrm{GH}$ & $311 \pm 247$ & $444 \pm 194^{*}$ & $434 \pm 208^{*}$ & $309 \pm 138$ & $301 \pm 142$ \\
\hline & GI & $242 \pm 157$ & $364 \pm 224^{*}$ & $354 \pm 181^{*}$ & $204 \pm 87$ & $261 \pm 78$ \\
\hline & GS & $169 \pm 73$ & $307 \pm 172^{*}$ & $300 \pm 122^{*}$ & $245 \pm 128$ & $205 \pm 117$ \\
\hline \multirow[t]{3}{*}{$\mathrm{DNa}\left(\mathrm{ml} \cdot \mathrm{min}^{-1}\right)$} & $\mathrm{GH}$ & $2,23 \pm 1,77$ & $3,20 \pm 1,40^{*}$ & $3,13 \pm 1,50^{*}$ & $2,25 \pm 1,01$ & $2,21 \pm 1,05$ \\
\hline & $\mathrm{GI}$ & $1,73 \pm 1,09$ & $2,64 \pm 1,58^{*}$ & $2,57 \pm 1,28^{*}$ & $1,49 \pm 0,61$ & $1,88 \pm 1,30$ \\
\hline & GS & $1,19 \pm 0,52$ & $2,19 \pm 1,22^{*}$ & $2,17 \pm 0,90^{\star}$ & $1,79 \pm 0,94$ & $1,50 \pm 0,86$ \\
\hline \multirow[t]{3}{*}{ EFNa (\%) } & $\mathrm{GH}$ & $4,00 \pm 3,80$ & $6,58 \pm 7,22^{*}$ & $5,10 \pm 3,48$ & $3,76 \pm 1,90$ & $3,38 \pm 1,66$ \\
\hline & $\mathrm{GI}$ & $2,99 \pm 3,43$ & $5,37 \pm 6,01^{*}$ & $5,50 \pm 5,99^{*}$ & $3,18 \pm 2,34$ & $2,55 \pm 1,77$ \\
\hline & GS & $1,76 \pm 1,19$ & $2,57 \pm 1,09$ & $2,88 \pm 1,84$ & $2,08 \pm 0,93$ & $4,00 \pm 4,31$ \\
\hline
\end{tabular}

$\# p<0,05$ : diferença significante entre os grupos em relação ao $\mathrm{GH}$

${ }^{*} p<0,05$ : diferença significante em relação ao momento controle do mesmo grupo 
Tabela IV - Valores da Depuração de Para-Aminohipurato de Sódio (DPAH), Depuração de Creatinina (DCr), Fração de Filtração (FF), Sódio Plasmático (PNa), Osmolalidade Plasmática (Posm) nos Momentos dos Grupos Estudados (Média \pm DP)

\begin{tabular}{|c|c|c|c|c|c|c|}
\hline \multirow[b]{2}{*}{ Atributos } & \multirow[b]{2}{*}{ Grupos } & \multicolumn{5}{|c|}{ Momentos } \\
\hline & & Controle & Ao15 & Ao30 & DAo15 & DAo30 \\
\hline \multirow[t]{3}{*}{ DPAH $\left(\mathrm{ml} \cdot \mathrm{min}^{-1} \cdot \mathrm{kg}^{-1}\right)$} & $\mathrm{GH}$ & $13,6 \pm 9,4$ & $11,7 \pm 7,1$ & $10,6 \pm 5,4$ & $8,2 \pm 3,4$ & $10,3 \pm 9,0$ \\
\hline & $\mathrm{GI}$ & $15,4 \pm 15,6$ & $15,7 \pm 18,8$ & $10,6 \pm 7,8$ & $11,2 \pm 12,7$ & $15,6 \pm 19,9$ \\
\hline & GS & $14,6 \pm 8,6$ & $15,9 \pm 16,9$ & $14,3 \pm 16,6$ & $13,0 \pm 13,8$ & $8,4 \pm 5,9^{*}$ \\
\hline \multirow[t]{3}{*}{$\mathrm{DCr}\left(\mathrm{ml} \cdot \mathrm{min}^{-1} \cdot \mathrm{kg}^{-1}\right)$} & $\mathrm{GH}$ & $3,6 \pm 1,2$ & $4,0 \pm 2,7$ & $3,9 \pm 2,4$ & $3,9 \pm 2,4$ & $4,0 \pm 2,0$ \\
\hline & $\mathrm{GI}$ & $5,3 \pm 4,9$ & $4,4 \pm 3,8$ & $4,2 \pm 2,7$ & $3,3 \pm 1,7$ & $4,6 \pm 2,5$ \\
\hline & GS & $4,1 \pm 1,6$ & $4,5 \pm 2,3$ & $5,5 \pm 4,4$ & $5,2 \pm 3,5$ & $3,5 \pm 2,3$ \\
\hline \multirow[t]{3}{*}{ FF } & $\mathrm{GH}$ & $0,35 \pm 0,16$ & $0,39 \pm 0,14$ & $0,38 \pm 0,11$ & $0,48 \pm 0,20$ & $0,47 \pm 0,17$ \\
\hline & $\mathrm{GI}$ & $0,38 \pm 0,21$ & $0,38 \pm 0,15$ & $0,45 \pm 0,19$ & $0,45 \pm 0,29$ & $0,44 \pm 0,24$ \\
\hline & GS & $0,37 \pm 0,19$ & $0,54 \pm 0,48$ & $0,63 \pm 0,44^{*}$ & $0,62 \pm 0,36^{*}$ & $0,63 \pm 0,44^{*}$ \\
\hline \multirow{2}{*}{$\mathrm{PNa}\left(\mathrm{mEq} \cdot \mathrm{L}^{-1}\right)$} & GI & $139 \pm 6$ & $137 \pm 11$ & $137 \pm 9$ & $136 \pm 9$ & $139 \pm 2$ \\
\hline & GS & $141 \pm 2$ & $140 \pm 2$ & $139 \pm 3$ & $139 \pm 3$ & $138 \pm 2$ \\
\hline \multirow[t]{3}{*}{ Posm (mOsm. kg ${ }^{-1} \mathrm{H}_{2} \mathrm{O}$ ) } & $\mathrm{GH}$ & $291 \pm 24$ & $285 \pm 58$ & $287 \pm 26$ & $283 \pm 33$ & $283 \pm 25$ \\
\hline & GI & $293 \pm 26$ & $295 \pm 15$ & $287 \pm 35$ & $295 \pm 16$ & $299 \pm 14$ \\
\hline & GS & $289 \pm 18$ & $294 \pm 12$ & $287 \pm 21$ & $285 \pm 19$ & $281 \pm 26$ \\
\hline
\end{tabular}

* $p<0,05$ : em relação ao momento controle do mesmo grupo

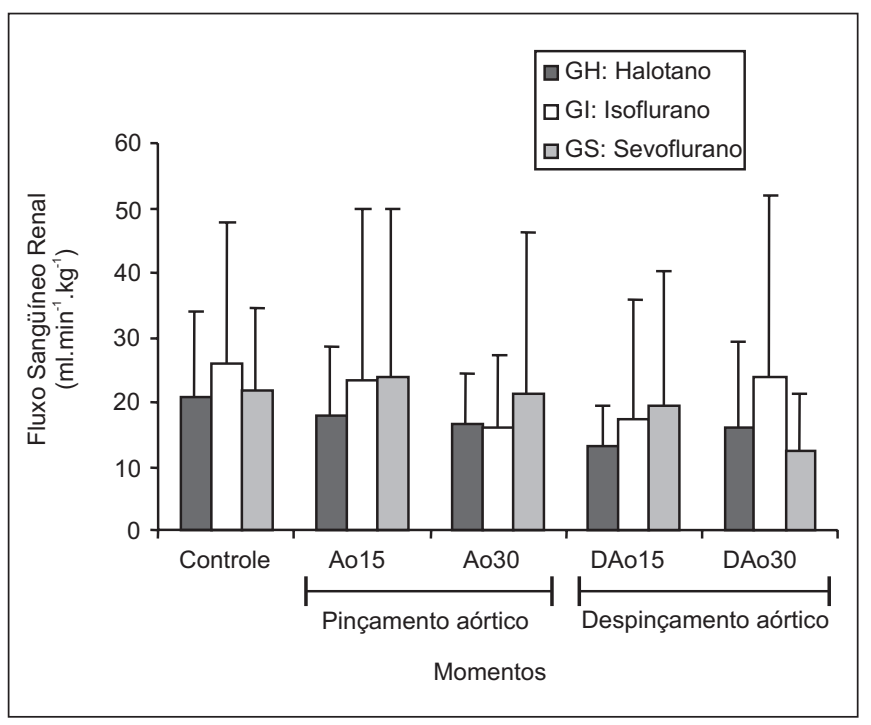

Figura 2 - Fluxo Sangüíneo Renal (FSR). Valores Obtidos nos Momentos dos Grupos Estudados com Indicação do Resultado Estatístico Obtido (Média \pm DP)

${ }^{*} p<0,05$ : GS: $($ Controle = Ao15) $>$ DAo30

Os valores do pHa diminuíram significantemente após o pinçamento aórtico em GH e Gl e após o despinçamento aórtico em GH, Gl e GS, enquanto os valores da $\mathrm{PaCO}_{2}$ e do $\mathrm{Ht}$ não se alteraram significantemente entre os grupos (Tabela V). Já os valores da $\mathrm{T}_{\text {Esof }}$ diminuíram significantemente em todos os grupos ao longo do experimento (Tabela V).

\section{DISCUSSÃO}

O presente estudo conduzido em animais aparentemente sadios, nos quais manteve-se a volemia, confirmou que o pinçamento aórtico determina alterações hemodinâmicas, mesmo quando realizado em níveis inferiores da aorta, como o pinçamento infra-renal. Essas alterações manifestaram-se, nos três grupos estudados, pela elevação da pressão arterial e das pressões de enchimento, como a doátrio direito e do índice sistólico. Assim, parece ter ocorrido redistribuição do volume sangüíneo da vasculatura distal para a vasculatura proximal à oclusão aórtica, com aumento da pré-carga.

Vários autores observaram aumento das pressões de enchimento durante o pinçamento aórtico ${ }^{27-29}$, enquanto outros autores não observaram essas alterações ${ }^{30}$. Por outro lado, muitos autores atribuem, como causa principal do aumento da pressão arterial média durante o pinçamento aórtico, o aumento súbito da impedância do fluxo aórtico, com aumento da pós-carga ${ }^{31}$, acompanhado, muitas vezes, por diminuição do índice cardíaco ${ }^{27}$. Na presente pesquisa, o índice de resistência vascular sistêmica não se alterou significantemente em nenhum dos grupos estudados, durante o pinçamento aórtico.

Quintin e col. ${ }^{29}$ observaram, no homem, recolhimento venoso passivo distal ao pinçamento aórtico, com liberação de catecolaminas (adrenalina e noradrenalina) e de angiotensina. A liberação dessas substâncias determinou vasoconstrição tanto proximal quanto distal ao pinçamento, com diminuição 
Tabela V - Valores do pH Arterial (pHa), Pressão Parcial de Dióxido de Carbono Arterial $\left(\mathrm{PaCO}_{2}\right)$, Hematócrito (Ht) e Temperatura Esofágica ( $T_{\text {Esof }}$ ) nos Momentos dos Grupos Estudados (Média $\pm D P$ )

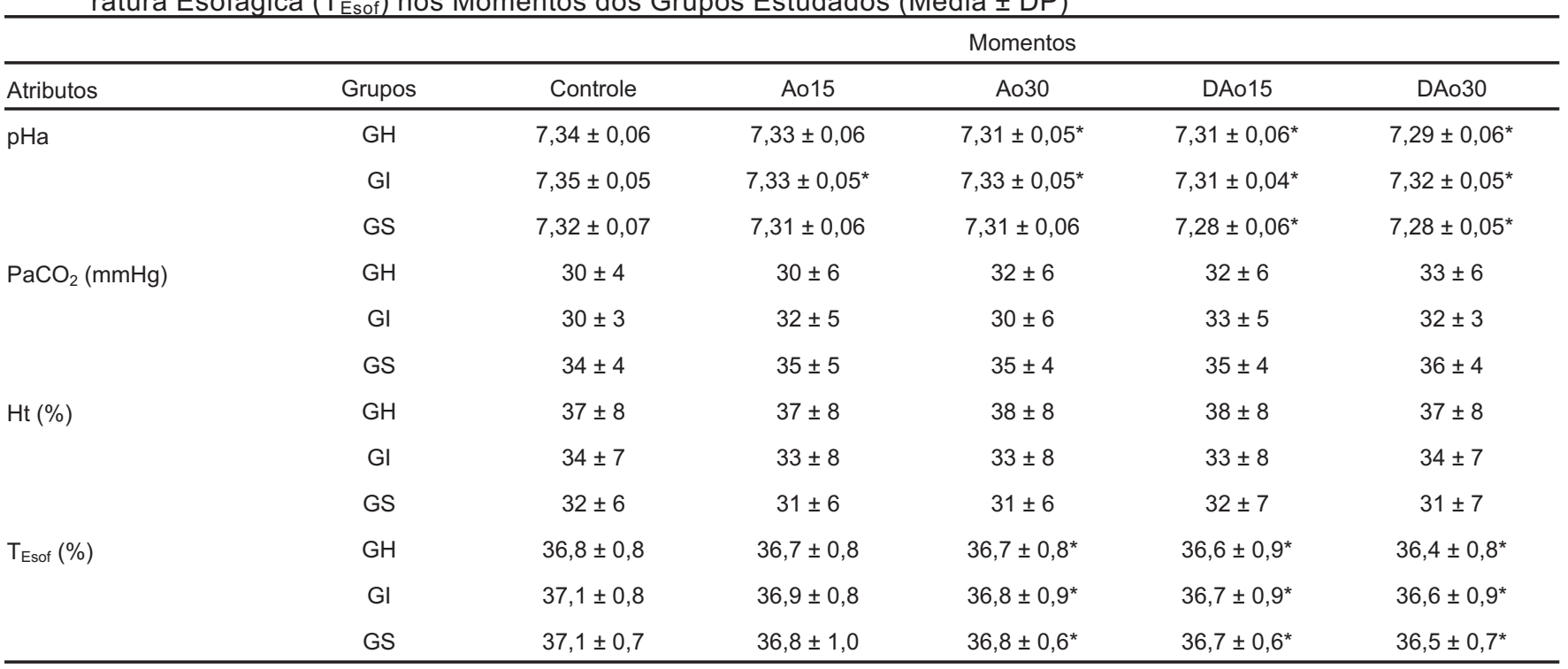

* $p<0,05$ em relação ao momento controle do mesmo grupo

do sistema de capacitância e deslocamento do volume sangüíneo proximal ao pinçamento, com grande interferência no retorno venoso, ou seja, na pré-carga, na dependência do nível de pinçamento aórtico ser supra ou infra-celíaco. Na primeira situação, o retorno venoso aumentou sempre, porque o deslocamento do volume sangüíneo ocorreu em direção aos músculos proximais ao pinçamento, aos pulmões e ao cérebro. Já no infra-celíaco, houve deslocamento do volume sangüíneo em direção aos órgãos esplâncnicos ou para outros tecidos proximais ao pinçamento. Caso o tônus venoso esplâncnico esteja diminuído, haverá diminuição do retorno venoso, mas se estiver elevado, haverá aumento do retorno venoso. Como conseqüência, a distribuição de sangue entre a vasculatura esplâncnica e a não esplâncnica é que irá determinar as alterações da pré-carga.

As repercussões renais do pinçamento aórtico foram caracterizadas por aumento da diurese e da natriurese, e diminuição da osmolaridade urinária com os três halogenados, enquanto com o sevoflurano houve aumento da resistência vascular renal, mas sem alteração significativa do ritmo de filtração glomerular e do fluxo plasmático renal, porém com aumento da fração de filtração.

A literatura apresenta resultados conflitantes em relação às repercussões do pinçamento aórtico infra-renal sobre a função renal, tanto em estudos experimentais ${ }^{32,33}$ como no homem $^{4,5}$. No entanto, os resultados mais observados têm sido o de aumento da resistência vascular renal ${ }^{30,32}$ e diminuição do fluxo sangüíneo renal ${ }^{4,5}$.

Dois fatores podem ter-se associado na possível gênese das alterações sobre a função renal encontradas na presente pesquisa: o primeiro refere-se à ação do pinçamento aórtico e o segundo, à ação do agente anestésico. Aoclusão da aorta infra-renal pode estar associada com aumento da resistên- cia vascular renal e moderado decréscimo no fluxo sangüíneo renal. Estas alterações hemodinâmicas podem persistir após a remoção do pinçamento ${ }^{34}$ por redução da filtração glomerular e do débito urinário. Há propensão do fluxo sangüíneo da córtex mais externa decrescer mais do que na parte mais interna da córtex, podendo causar redistribuição do fluxo sangüíneo dentro dos rins, após a aplicação do pinçamento aórtico oclusivo ${ }^{3,6}$. A má distribuição do fluxo sangüíneo no interior dos rins pode persistir por até 60 minutos após o despinçamento infra-renal da aorta ${ }^{4}$.

Colson e col. ${ }^{8}$ estudaram, em pacientes submetidos a pinçamento aórtico infra-renal, os efeitos renais da anestesia com isoflurano e halotano. Durante o período de pré-pinçamento, o fluxo plasmático renal e a filtração glomerular foram significativamente menores nos pacientes que receberam halotano em relação aos que receberam isoflurano. Durante o pinçamento, os atributos renais não foram afetados de forma marcante nos dois grupos, mas a filtração glomerular permaneceu mais elevada nos pacientes sob isoflurano. Após o despinçamento, os atributos renais normalizaram-se, inclusive no grupo do halotano. Este estudo mostrou que a anestesia com halotano associa-se com alterações renais importantes, antes e durante o pinçamento aórtico, o que sugere, segundo os autores, a ocorrência de vasoconstrição renal importante. Com o isoflurano, ao contrário, houve melhora na hemodinâmica renal. Como os atributos hemodinâmicos estudados antes, durante e após o pinçamento aórtico não diferiram entre os dois anestésicos, os autores concluem que o benefício observado na função renal nos pacientes que receberam isoflurano não pode ser atribuído aos efeitos do isoflurano na hemodinâmica sistêmica, mas aos seus efeitos renais.

Em outro estudo, Colson e col. ${ }^{12}$ verificaram em pacientes submetidos a pinçamento aórtico infra-renal sob anestesia 
com isoflurano, halotano, ou droperidol e flunitrazepam, que o isoflurano preveniu as alterações renais que ocorreram com o pinçamento aórtico, ou seja, diminuição do ritmo de filtração glomerular e do volume urinário, o que não aconteceu com o halotano, flunitrazepam e droperidol. A piora da função renal, já no período pré-pinçamento, no grupo que utilizou halotano, sugere que ocorreu vasoconstrição renal intensa, que pode ter sido mediada pelo sistema nervoso simpático. Já as ações do isoflurano de vasodilatação e de inibição da atividade da renina podem ter contribuído para os resultados obtidos.

Sempre que se realiza expansão do volume extracelular, com solução fisiológica, como na presente pesquisa, haverá aumento da diurese e diminuição da osmolalidade urinária. Isto acontece porque o rim, visando incrementar a eliminação do volume excedente, aciona um sistema multifatorial complexo que envolve diferentes vias neuro-humorais ${ }^{35}$. Assim, durante o pinçamento aórtico infra-renal, a associação da expansão volêmica com o aumento da pré-carga pode ter provocado distensão atrial. Em resposta ao estiramento da parede, os miócitos atriais elevam a secreção do fator atrial natriurético, que além de causar vasodilatação sistêmica, atua em receptores específicos no rim, aumenta a excreção renal de sódio e água, ao inibir a reabsorção tubular de sódio, e aumenta o ritmo de filtração glomerular ${ }^{36}$. O fator natriurético também pode diminuir a pressão arterial, ao relaxar a musculatura lisa vascular, e a estimulação simpática, e inibir o sistema renina-angiotensina-aldosterona e a liberação de hormônio antidiurético.

Somando-se a isto, existem, no átrio, receptores neurais que respondem ao estiramento e à alterações na pressão transmural. Há dois tipos de mecanorreceptores: os do tipo A, que se localizam próximo à entrada das veias de grande calibre e que não sofrem influência de variações do volume do átrio, e os do tipo B, que são ativados com o enchimento atrial. Os sinais de estiramento e tensão são detectados e conduzidos pelos IX eX pares cranianos aos centros medulares e hipotalâmicos, determinando respostas fisiológicas integradas, dentre as quais a inibição da liberação do hormônio antidiurético ${ }^{37}$

O hormônio antidiurético, cuja ação principal se faz sobre os túbulos distais e coletores, aumentando a permeabilidade à água, é liberado quando há variação na osmolaridade e no volume do meio externo. Um outro estímulo para produção do hormônio antidiurético é o decorrente da ação da angiotensina II, formada a partir da secreção de renina em áreas circundantes ao $3^{\circ}$ ventrículo. A angiotensina II, além de potente vasoconstritor com ação direta na musculatura lisa dos vasos, atua na supra-renal, aumentando a produção de aldosterona, cuja ação principal é aumentar a reabsorção de sódio e água no segmento cortical coletor.

A maioria dos autores tem observado aumento de atividade do sistema renina-angiotensina durante o pinçamento aórtico, no cão ${ }^{38}$ e no homem ${ }^{39-41}$. O aumento da atividade da renina pode ser facilmente explicado no pinçamento torácico ou supra-renal da aorta, quando ocorre diminuição da pressão de perfusão das arteríolas aferentes renais. Contudo, o aumento da atividade da renina, quando do pinçamento da aorta infra-renal, não encontra explicações claras na literatura, podendo decorrer em conseqüência do próprio pinçamento aórtico, por efeito da técnica anestésica empregada ou por alterações de volume ${ }^{9}$.

Na presente pesquisa, a possibilidade de que tenha ocorrido aumento da atividade hormonal em decorrência do pinçamento aórtico, principalmente do sistema renina-angiotensina-aldosterona e do hormônio antidiurético, fica prejudicada, em razão da resposta cardiovascular e das alterações renais encontradas, com aumento do volume urinário e das excreções urinária e fracionária de sódio, além de diminuição da osmolaridade urinária, sem que houvesse aumento da resistência vascular renal nos grupos do halotano e isoflurano. Por outrolado, não pode ficar inteiramente afastada no grupo de sevoflurano, principalmente de ativação do sistema renina-angiotensina-aldosterona, por causa do aumento da resistência vascular renal e da fração de filtração, e ausência de efeito significante na excreção fracionária de sódio.

Ma e col. ${ }^{42}$ verificaram que o sevoflurano determinou, em nervos renais do cão, maior depressão (dose-dependente) dos reflexos somatossimpáticos mediados pela fibra $C$ (de condução lenta) do que pelas fibras A delta (de condução rápida), e somente deprime a atividade simpática renal espontânea em concentrações superiores a 3\%. Essas ações parecem ser semelhantes às determinadas pelo isoflurano, mas diferente das determinadas pelo halotano que deprime na mesma proporção os reflexos somatossimpáticos mediados pelas fibras $\mathrm{C}$ e A delta com efeitos insignificantes sobre a atividade simpática espontânea, e também diferentes das do desflurano, que em baixas concentrações é excitatório e em elevadas concentrações inibe igualmente tanto os reflexos mediados pelas fibras $\mathrm{A}$ delta e $\mathrm{C}$ como a atividade simpática renal espontânea ${ }^{43}$.

Esta ação diferenciada dos anestésicos halogenados sobre a atividade espontânea e reflexa simpática dos nervos renais frente a um determinado estímulo, geralmente algogênico, pode ajudar na compreensão dos resultados que se obtiveram em relação à vasoconstrição renal observada com o sevoflurano durante o pinçamento e após o despinçamento aórtico infra-renal.

Teoricamente, os anestésicos halogenados que promovem vasodilatação, como o isoflurano e sevoflurano, apresentariam vantagens em cirurgias de reconstrução aórtica, evitando que ocorresse durante o pinçamento aórtico aumento da resistência vascular sistêmica e renal. Assim, praticamente já existe consenso da boa indicação do isoflurano em cirurgias vasculares. No entanto, para o sevoflurano ainda não existe uma situação bem definida nas cirurgias de reconstrução aórtica. Pelos resultados obtidos, com manutenção da resistência vascular renal em valores mais elevados do que os do controle durante e após o pinçamento, e diminuição dos fluxos plasmático e sangüíneo renais após o despinçamento, a sua indicação nesse tipo de cirurgia vascular nos parece ser bem mais limitada do que a do isoflurano ou halotano. Entretanto, mais estudos são necessários para se verificar a indicação em cirurgia de reconstrução aórtica. Em re- 
lação ao halotano, os resultados surpreenderam, pois os autores têm encontrado importantes alterações renais com esse halogenado, seja na fase de controle ou durante o pinçamento aórtico. Provavelmente o uso de baixas concentrações dos halogenados na presente pesquisa deve ter influenciado os resultados obtidos.

Apesar de não terem sido utilizados nesse estudo marcadores específicos de alterações tubulares renais, não há evidências de que tenham ocorrido alterações renais durante o momento controle do estudo, pois os testes de função glomerular e tubulares utilizados estão dentro dos parâmetros normais para o cão. Além disso, a limitação da concentração do sevoflurano em 0,75 CAM e a utilização de fluxos médios de gases frescos por período limitado de 3 horas foram precauções para que o níveis de íons fluoretos e do composto Anão alcançassem níveis elevados. Ao se limitar a concentração dos halogenados em 0,75 CAM também se preveniu a ocorrência de alterações hemodinâmicas e renais ainda mais importantes que, provavelmente, são dose-dependentes.

Embora tenha sido tomado cuidado com a temperatura ambiente, que permaneceu entre 24 e $25^{\circ} \mathrm{C}$, e tenham-se recoberto os cães com campos cirúrgicos, houve diminuição da temperatura esofágica, em todos os grupos, ocasionando hipotermia de grau leve a moderado nos cães, pois a temperatura central na espécie é de 38 a $39^{\circ} \mathrm{C}^{44}$. Certamente, o fato de ter-se utilizado solução glicosada a $5 \%$ gelado como indicador para medida do débito cardíaco, pode também ter colaborado para o desenvolvimento de hipotermia nos animais, pois cada resultado consistiu de três medidas consecutivas, utilizando-se, assim, $30 \mathrm{ml}$ do indicador em cada momento estudado, o que totalizou a infusão de $150 \mathrm{ml}$ de solução glicosada gelada durante a experimentação. Apesar de sua instalação, a intensidade da hipotermia certamente não foi suficiente para alterar os resultados obtidos na pesquisa. Durante e após o pinçamento aórtico houve, em todos os grupos, a ocorrência de acidose metabólica, que provavelmente foi devida à isquemia dos tecidos abaixo do pinçamento. Concluindo, no cão e nas condições experimentais empregadas, o halotano e o isoflurano atenuaram a principal alteração que ocorre durante o pinçamento aórtico infra-renal, que é o aumento da resistência vascular renal, o que não ocorreu com o sevoflurano.

Revista Brasileira de Anestesiologia

Vol. 53, N 6, Novembro - Dezembro, 2003

\section{Effects of Halothane, Isoflurane and Sevoflurane on Renal Function in Dogs under Infra-Renal Aortic Cross-Clamping}

Flora Margarida Barra Bisinotto, TSA, M.D.; José Reinaldo Cerqueira Braz, TSA, M.D.

\section{INTRODUCTION}

Surgical aortic aneurysm correction, even in the infra-renal segment, may be associated to approximately $5 \%$ renal failure, in general due to acute tubular necrosis, which normally requires hemodialysis ${ }^{1}$ and has a high mortality rate of approximately $50 \%$, in spite of all advances in renal failure diagnosis and management ${ }^{2}$. When the surgery is performed in the supra-renal aortic segment, renal dysfunction incidence may reach $15 \%$ to $30 \%$, and when it requires chest segment occlusion the incidence may reach $50 \%{ }^{3}$.

Infra-renal aortic cross-clamping may induce approximately $30 \%$ renal vascular resistance increase and renal blood flow decrease, which may persist after aortic unclamping ${ }^{4}$, and even for some months after surgery ${ }^{5}$. When infra-renal aorta is unclamped, there is in general blood flow redistribution to kidneys, with cortical renal blood flow and glomerular filtration rate decrease ${ }^{6,7}$, which seem to be unrelated to cardiac output or mean blood pressure changes ${ }^{7,8}$, but rather to renal vascular resistance increase ${ }^{9}$. Urinary volume decrease that may occur seems to be unrelated to glomerular filtration rate decrease ${ }^{7}$ or to postoperative renal failure severity ${ }^{10}$.

Several factors have contributed for decreased mortality during surgical abdominal aneurysm correction, such as improved surgical technique, earlier interventions, better patients selection, monitoring equipments, new drugs and anesthetic techniques, and improved postoperative intensive care $^{9}$

Anesthetics may play an important role in the pathogenesis of cardiac and renal changes during aortic cross-clamping, for their effects on cardiovascular hemodynamics and hormones release. So, halothane seems to be associated to more renal changes as compared to isoflurane in equipotent doses in patients submitted to surgical aortic reconstruction under infra-renal aortic cross-clamping ${ }^{11,12}$, probably for decreasing cardiac output, renal blood flow and glomerular filtration rate in a higher rate as compared to isoflurane. According to the authors ${ }^{11,12}$, these results suggest that anesthesia with halothane is associated to renal vasoconstriction, which is not present with isoflurane. As to sevoflurane, early studies have not found major renal changes, especially in renal concentration capacity, both in men ${ }^{13,14}$ and experimental animals ${ }^{15,16}$. But the concern with renal concentration function is relevant because metabolized sevoflurane forms fluorine ions, which potentially cause renal tubular injury. There is also the concern with the formation of fluormethyl -2,2- difluorine - 1 - (trifluormethyl) vinyl ether, also known as compound $A$, because sevoflurane may form metabolic products in the presence of carbon dioxide absorbers, by 
action of renal $\beta$-liase enzyme. This substance is nefrotoxic in rats $^{17,18}$, but not in men, due to the lower $\beta$-liase activity in the latter. But compound $\mathrm{A}$ is also formed in low concentrations in anesthetic circuits, even closed circuits, using low fresh gases flow. So, it seems to have no nefrotoxic effects in men ${ }^{19}$.

Some authors have recently observed that sevoflurane in low fresh gases flow may determine renal changes, characterized by increased albumin and glucose urinary excretion, as well as of some tubular enzymes ${ }^{20,21}$. These results, however, have been questioned by other authors ${ }^{14,22,23}$ for believing that the best renal changes markers are still plasma urea and creatinine levels, and for the inexistence of significant evidences to credit tubular enzyme changes as major indicators of sevoflurane-induced renal injury. On the other hand, the authors have not observed renal function changes in patients with stable renal failure submitted to elective surgery under sevoflurane or isoflurane ${ }^{23,24}$.

Based on literature results, the FDA has not approved the use of sevoflurane in the presence of fresh gases flow equal to or lower than 1 liter.

There are no studies in the literature on sevoflurane effects on the renal system in surgeries which may determine renal changes, such as those which may be present during surgical aortic reconstruction.

This study aimed at comparing halothane, isoflurane and sevoflurane effects, in equipotent doses, on the renal function of dogs submitted to infra-renal aortic cross-clamping, to determine which inhalational agents better "protect" kidneys against aortic cross-clamping and unclamping effects.

\section{METHODS}

After the Animal Research Ethics Committee approval, 30 adult dogs of both genders, weighing 18 to $20 \mathrm{~kg}$ were involved in the study. Dogs were randomly distributed in 3 groups of 10 animals each. The experiment had two phases:

$1^{\text {st }}$ phase: anesthetic induction, tracheal intubation, artificial ventilation, anesthetic maintenance with halothane, isoflurane or sevoflurane, depending on the studied group, monitoring of ventilation, oxygenation, cardiovascular hemodynamics, renal function and temperature, followed by median laparoscopy to prepare infra-renal aortic cross-clamping;

$2^{\text {nd }}$ phase: aortic cross-clamping for 30 minutes, followed by aortic unclamping.

Groups were divided as follows:

$\mathrm{GH}$ - Anesthetic maintenance with $0.67 \%$ halothane $(0.75$ $\mathrm{MAC}$ );

$\mathrm{GI}$ - Anesthetic maintenance with $0.96 \%$ isoflurane $(0.75$ MAC);

GS - Anesthetic maintenance with $1.8 \%$ sevoflurane $(0.75$ $\mathrm{MAC})$.

MAC was calculated according to Kasama and lkeda's proposition (1988) ${ }^{25}$.

\section{Experimental Sequence}

After 12-hour fast, but with free access to water, anesthesia was induced with intravenous propofol $\left(5.5 \mathrm{mg}^{\mathrm{kg}}{ }^{-1}\right)$ and alcuronium $\left(0.2 \mathrm{mg} \cdot \mathrm{kg}^{-1}\right)$ and animals were placed on Claude Bernard device in the experimental room where the temperature was maintained by air conditioning (warm/cold) between 24 and $25^{\circ}$. The following procedures were then performed:

1. Tracheal intubation with 8.5 to $9 \mathrm{~mm}$ internal diameter, high compliance cuff tracheal tube, and volume controlled ventilation with ventilator Ohmeda (USA) of the anesthesia machine Excel mod. 210 SE (Ohmeda USA) and compressed air (1.2 L. $\left.\mathrm{min}^{-1}\right)$ and oxygen (0.8 L. $\mathrm{min}^{-1}$ ) flow in a semi-closed circle system with carbon dioxide reabsorber (soda lime). Oxygen inspired fraction was maintained in $40 \%$ to $50 \%$, tidal volume in $20 \mathrm{ml}^{\mathrm{kg}} \mathrm{kg}^{-1}$ and respiratory rate in 10 to $15 \mathrm{mov}^{\mathrm{min}} \mathrm{m}^{-1}$ to maintain end $\mathrm{CO}_{2}$ expiratory pressure in 30 to $35 \mathrm{mmHg}$;

2. Installation of Datex-Engstron (Finland) AS3 device for reading and recording of ventilatory parameters, anesthetic gases, hemodynamic parameters, oxygenation and temperature;

3. Halogenate agent was inhaled via gaged vaporizer specific to each halogenate (Ohmeda, USA). $\mathrm{O}_{2}$ and $\mathrm{CO}_{2}$ inspired and expired concentration of each agent was controlled by AS3 device Gases Analyzer (Datex-Engstron - Finland), using gases sample capturer close to the "Y" valve of the respiratory circuit. Initial MAC $(0.89 \%$ halothane, $1.3 \%$ isoflurane and $2.4 \%$ sevoflurane) was determined according to expired concentration;

4. Installation of three-channel ECG ( $D_{\|}$lead), thermometer sensor on the lower third of the esophagus and forceps-shaped sensor placed on animals' tongue for oxygen peripheral saturation reading;

5. Right femoral vein dissection and catheterization for drug administration, such as additional alcuronium doses $\left(0.06 \mathrm{mg} \cdot \mathrm{kg}^{-1}\right)$, and venous blood collection;

6 . Left femoral vein dissection and catheterization for lactated Ringer's continuous infusion $\left(18 \mathrm{ml} . \mathrm{kg}^{-1}\right)$. Priming creatinine (3\%) and para-aminophuric acid (PAH) $(0.4 \%)$ doses were administered 30 minutes after, using 1 $\mathrm{ml} . \mathrm{kg}^{-1}$ of the solution. Then, Ringer's solution (18 $\left.\mathrm{ml} . \mathrm{kg}^{-1}\right)$ with PAH $(0.08 \%)$ and creatinine $(0.2 \mathrm{~g} \%)$ were started, being administered $0.6 \mathrm{mg} \cdot \mathrm{kg}^{-1}$ creatinine and $0.24 \mathrm{mg} . \mathrm{kg}^{-1} \mathrm{PAH}$ per minute until experiment completion, through two-channel infusion pump (Abbott's Anne USA);

7. Left femoral artery dissection and catheterization to measure aortic pressure and control aortic cross-clamping and unclamping;

8. Left axillary artery dissection and catheterization to measure mean blood pressure and collect blood for $\mathrm{pH}$ and blood gases analysis in Chiron Diagnostics device, mod. Rapidlab 865 (UK);

Revista Brasileira de Anestesiologia Vol. 53, № 6, Novembro - Dezembro, 2003 
9. Right external jugular vein dissection and catheterization with $8.5 \mathrm{~F}$ introducer and insertion of 7F Swan-Ganz catheter until the pulmonary vein to measure pressures and cardiac output by thermodilution;

10. Vesical catheterization to collect urine in graduated pipettes;

11. Median laparoscopy and infra-renal aorta dissection. Placement of cardiac tape around the aorta immediately after renal arteries emergence, for further aortic cross-clamping. Cardiac tape was trespassed in a small $15 \mathrm{~cm}$ plastic tube. Surgical incision was then closed around the plastic tube;

12. After preparation, halogenates MAC was decreased, according to the studied group, from 1 to $0.75 \mathrm{MAC}$, and 30-minutes hemodynamic stabilization period was started;

13. Attributes measurement and blood (arterial and venous) and urine collection (control moment);

14. Intravenous $70 \mathrm{UI} . \mathrm{kg}^{-1}$ heparin injection and infra-renal aortic cross-clamping 3 minutes later, advancing the plastic tube along the cardiac tape until there was no femoral artery pressure recording;

15. Attributes measurement and blood and urine collection 15 and 30 minutes after aortic cross-clamping (moments Ao15 and Ao30);

16. Aortic unclamping, attributes measurement and blood and urine collection 15 and 30 minutes after aortic unclamping (moments DAo15 and DAo30);

17. End of experiment and animals sacrifice with a sodium pentobarbital overdose.

\section{Studied Attributes}

Attributes studied were:

Anthropometric - weight (kg), length ( $\mathrm{cm}$ ) and body surface $\left(\mathrm{m}^{2}\right)$; Gender; Hemodynamic - heart rate (HR), mean blood pressure (MBP), mean right atrium pressure (RAP), mean pulmonary artery pressure (PAP), mean pulmonary capillary wedge pressure (PCWP), cardiac index $(\mathrm{Cl})$, systolic index (SI) and systemic vascular resistance index (SVRI); Renal function - sodium para-aminophurate clearance (PAHD) to measure renal plasma flow (RPF), renal blood flow $(\mathrm{RBF}=\mathrm{RPF} / 1-\mathrm{Ht})$, renal vascular resistance $(\mathrm{RVR}=$ MBP $\times 80 / R B F \times 10^{-3}$ ), urinary output (UO), urinary osmolality (Uosm), creatinine clearance $(\mathrm{CrC})$ to determine glomerular filtration rate (GFR), filtration fraction $(\mathrm{FF}=\mathrm{CrC} / \mathrm{PAHD})$, osmolar clearance $(\mathrm{Cosm}=$ Uosm $\mathrm{x}$ UO/Posm), free water clearance $\left(\mathrm{DH}_{2} \mathrm{O}=\mathrm{UO}\right.$ - Dosm), sodium urinary excretion (UENa $=\mathrm{UO} \times \mathrm{UNa}$ ), sodium $\mathrm{N}$ clearance ( $\mathrm{CNa} \times \mathrm{UO} / \mathrm{PNa}$ ) and sodium fractional excretion ( $\mathrm{CNa} / \mathrm{CrC} \times 100)$; Blood - hematocrit $(\mathrm{Ht})$, plasma sodium (PNa), plasma osmolality (Posm), arterial $\mathrm{pH}(\mathrm{pHa})$, arterial $\mathrm{CO}_{2}$ partial pressure $\left(\mathrm{PaCO}_{2}\right)$; Metabolic - esophageal temperature $\left(\mathrm{T}_{\text {Eoph }}\right)$.
Statistical Analysis

Profile Analysis was used for statistical analysis of results ${ }^{26}$. ANOVA was used for anthropometric variables and Chi-square test $\left(\mathrm{X}^{2}\right)$ was used for gender distribution, considering significant $p<0.05$.

\section{RESULTS}

There were no significant differences among groups in anthropometric values and gender distribution (Table I).

Table I - Anthropometric Attributes and Gender Distribution within Groups

\begin{tabular}{lcccc}
\hline Groups & $\begin{array}{c}\text { Weight } \\
(\mathrm{kg})^{*}\end{array}$ & $\begin{array}{c}\text { Length } \\
(\mathrm{cm})^{*}\end{array}$ & $\begin{array}{c}\text { Body Surface } \\
\left(\mathrm{m}^{2}\right)^{*}\end{array}$ & $\begin{array}{c}\text { Gender } \\
(\mathrm{M} / \mathrm{F})\end{array}$ \\
\hline $\mathrm{GH}$ & $18.3 \pm 1.6$ & $101.2 \pm 7.8$ & $0.702 \pm 0.067$ & $7 / 3$ \\
$\mathrm{GI}$ & $19.9 \pm 2.0$ & $103.0 \pm 5.6$ & $0.731 \pm 0.043$ & $6 / 4$ \\
GS & $19.7 \pm 2.1$ & $104.1 \pm 5.2$ & $0.736 \pm 0.061$ & $8 / 2$ \\
\hline
\end{tabular}

There were no significant differences among groups $(p>0.05)$

* Values expressed in Mean \pm SD

Heart rate was the only hemodynamic parameter with significant changes among groups, with lower GH values as compared to other groups during aortic cross-clamping (Table II). There has been MBP, RAP and SI increase during aortic cross-clamping in all groups, while PAP has increased in $\mathrm{GH}$ and $\mathrm{GI}$, and $\mathrm{Cl}$ has increased in $\mathrm{GI}$ (Table II). Increased hemodynamic parameters have returned to baseline values after aortic unclamping, except for $\mathrm{Cl}$ and $\mathrm{SI}$, which have remained high (Table II). SVRI, which was not significantly changed during aortic cross-clamping in all groups, has significantly decreased in all groups.

Free water clearance was the only renal parameter with significant changes among groups, being $\mathrm{GI}$ and GS values lower as compared to $\mathrm{GH}$ after aortic unclamping. UO, UENa, and $\mathrm{CNa}$ were significantly increased in all groups during aortic cross-clamping, while Uosm was decreased (Table III). RVR was increased in GS (Figure 1), followed by FF increase (Table IV). FENa was increased in GH and GI (Table III). Increased renal parameters have returned to baseline values after aortic unclamping, except for Uosm which has remained in lower levels as compared to baseline values in all groups (Table III) and RVR (Figure 1) and FF (Table IV) which have remained significantly high in GS, followed by significant decrease in RBF and PAHC (Figures 2 and Table V, respectively. CrC was not changed in all experimental groups in all studied moments (Table IV).

$\mathrm{pHa}$ values were significantly decreased after aortic cross-clamping in $\mathrm{GH}$ and $\mathrm{GI}$, and after aortic unclamping in $\mathrm{GH}, \mathrm{GI}$ and $\mathrm{GS}$, while $\mathrm{PaCO}_{2}$ and $\mathrm{Ht}$ values were not significantly different among groups (Table $\mathrm{V}$ ). $\mathrm{T}_{\text {Esoph }}$ values were significantly decreased in all groups throughout the experiment (Table V). 
Table II - Heart Rate (HR), Mean Blood Pressure (MBP), Right Atrium Pressure (RAP), Pulmonary Artery Pressure (PAP), Pulmonary Capillary Wedge Pressure (PCWP), Cardiac Index (CI), Systolic Index (SI) and Systemic Vascular Resistance Index (SVRI) in Moments of Studied Groups (Mean \pm SD)

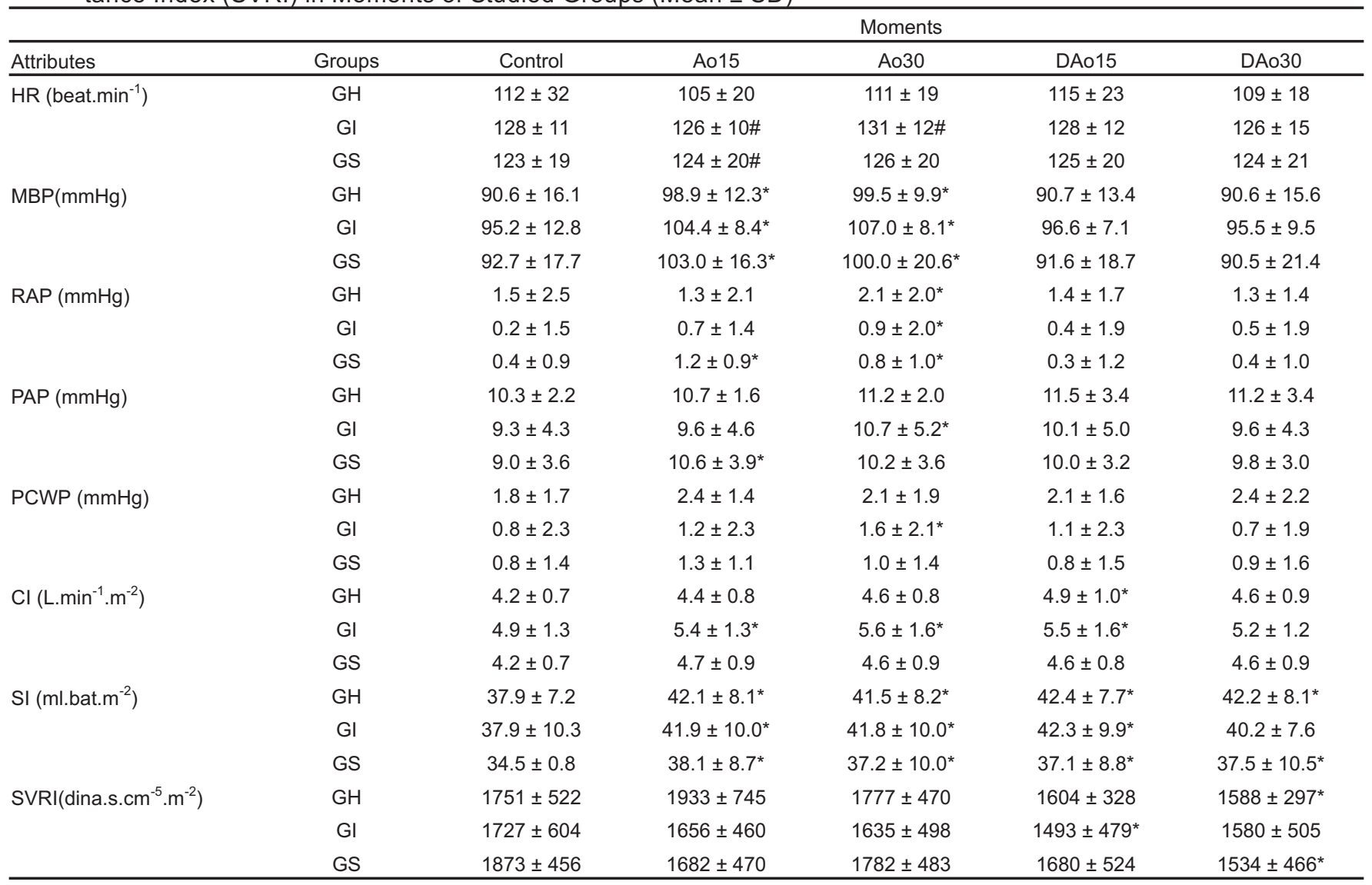

$\# \mathrm{p}<0.05$ : significant difference among groups as compared to $\mathrm{GH}$

${ }^{*} p<0.05$ : significant difference as compared to control moment of the same group

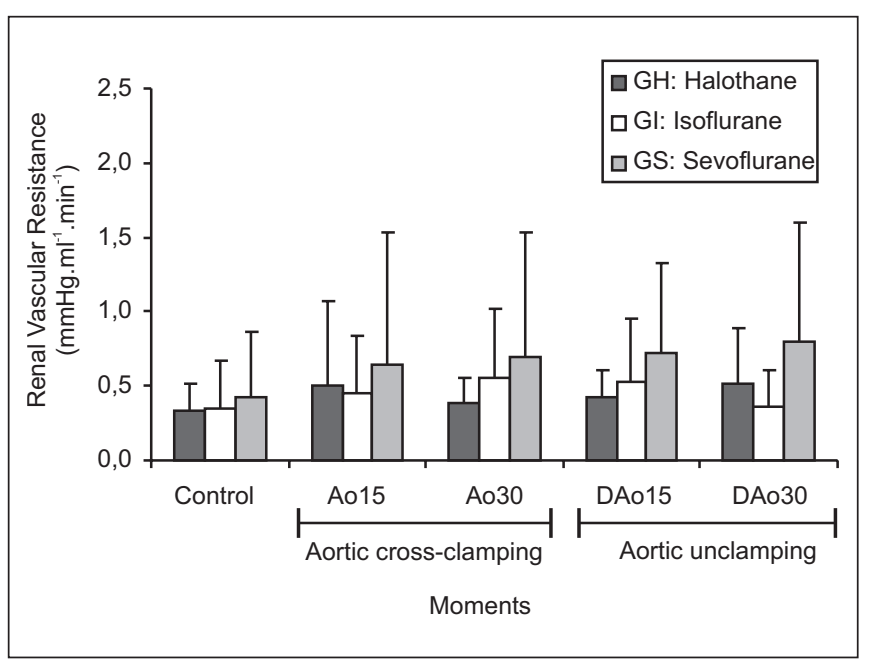

Figure 1 - Renal Vascular Resistance (RVR). Values obtained in Moments of Studied Groups with Indication of Statistical Result (Mean \pm SD)

${ }^{\star} p<0.05:$ GS: Control < (Ao30 = DAo15 = DAo30 $)$

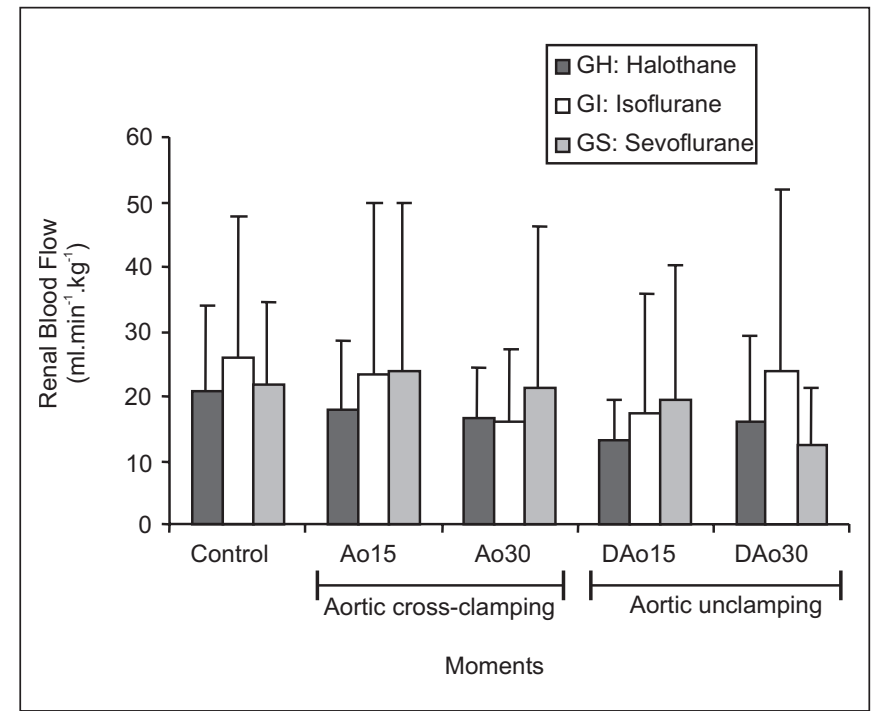

Figure 2 - Renal Blood Flow (RBF). Values obtained in Moments of Studied Groups with Indication of Statistical Result (Mean \pm $\mathrm{SD})$

${ }^{*} p<0.05:$ GS: $($ Control $=$ Ao15) $>$ DAo30

Revista Brasileira de Anestesiologia Vol. 53, N 6, Novembro - Dezembro, 2003 
Table III - Urinary Output (UO), Urinary Osmolarity (Uosm), Osmolar Clearance (Cosm), Free Water Clearance $\left(\mathrm{CH}_{2} \mathrm{O}\right)$, Sodium Urinary Excretion (UENa), Sodium Clearance ( $\mathrm{CNa}$ ) and Sodium Fractional Excretion (FENa) within Moments of Studied Groups (Mean \pm SD)

\begin{tabular}{|c|c|c|c|c|c|c|}
\hline \multirow[b]{2}{*}{ Attributes } & \multirow[b]{2}{*}{ Groups } & \multicolumn{5}{|c|}{ Moments } \\
\hline & & Control & Ao15 & Ao30 & DAo15 & DAo30 \\
\hline \multirow[t]{3}{*}{$\mathrm{UO}\left(\mathrm{ml} \cdot \mathrm{min}^{-1} \cdot \mathrm{kg}^{-1}\right)$} & $\mathrm{GH}$ & $0.14 \pm 0.11$ & $0.18 \pm 0.09^{*}$ & $0.19 \pm 0.09^{*}$ & $0.14 \pm 0.08$ & $0.14 \pm 0.06$ \\
\hline & $\mathrm{Gl}$ & $0.10 \pm 0.06$ & $0.14 \pm 0.07$ & $0.15 \pm 0.06^{*}$ & $0.09 \pm 0.04$ & $0.12 \pm 0.07$ \\
\hline & GS & $0.10 \pm 0.11$ & $0.16 \pm 0.13^{*}$ & $0.15 \pm 0.09^{*}$ & $0.13 \pm 0.08$ & $0.11 \pm 0.07$ \\
\hline \multirow[t]{2}{*}{ Uosm (mOsm. $\mathrm{kg}^{-1}$ of water) } & $\mathrm{GH}$ & $487 \pm 260$ & $378 \pm 157^{*}$ & $345 \pm 157^{*}$ & $370 \pm 182^{*}$ & $377 \pm 182^{*}$ \\
\hline & GS & $738 \pm 209$ & $564 \pm 172^{*}$ & $557 \pm 168^{*}$ & $577 \pm 147^{*}$ & $598 \pm 161^{*}$ \\
\hline \multirow[t]{3}{*}{$\operatorname{Cosm}\left(\mathrm{ml} \cdot \mathrm{min}^{-1}\right)$} & $\mathrm{GH}$ & $3.29 \pm 1.72$ & $4.24 \pm 1.80$ & $3.86 \pm 1.85$ & $3.00 \pm 1.17$ & $3.34 \pm 1.66$ \\
\hline & $\mathrm{Gl}$ & $4.41 \pm 2.72$ & $4.88 \pm 2.20$ & $4.65 \pm 2.13$ & $2.91 \pm 1.59$ & $3.57 \pm 2.11$ \\
\hline & GS & $4.33 \pm 3.20$ & $5.25 \pm 3.18$ & $5.13 \pm 2.73$ & $4.40 \pm 1.63$ & $4.09 \pm 1.57$ \\
\hline \multirow[t]{2}{*}{$\mathrm{CH}_{2} \mathrm{O}\left(\mathrm{ml} \cdot \mathrm{min}^{-1}\right)$} & $\mathrm{GH}$ & $-0.85 \pm 1.18$ & $-0.89 \pm 2.26$ & $-0.45 \pm 1.81$ & $-0.39 \pm 1.21$ & $-0.74 \pm 1.07$ \\
\hline & GS & $-2.28 \pm 1.32$ & $-2.05 \pm 1.60$ & $-2.22 \pm 1.96$ & $-1.89 \pm 0.53 \#$ & $-1.87 \pm 0.86$ \\
\hline \multirow[t]{3}{*}{ UENa $\left(\mu \mathrm{Eq} \cdot \mathrm{min}^{-1}\right)$} & $\mathrm{GH}$ & $311 \pm 247$ & $444 \pm 194^{*}$ & $434 \pm 208^{*}$ & $309 \pm 138$ & $301 \pm 142$ \\
\hline & $\mathrm{Gl}$ & $242 \pm 157$ & $364 \pm 224^{*}$ & $354 \pm 181^{*}$ & $204 \pm 87$ & $261 \pm 78$ \\
\hline & GS & $169 \pm 73$ & $307 \pm 172^{*}$ & $300 \pm 122^{*}$ & $245 \pm 128$ & $205 \pm 117$ \\
\hline \multirow[t]{3}{*}{$\mathrm{CNa}\left(\mathrm{ml} \cdot \mathrm{min}^{-1}\right)$} & $\mathrm{GH}$ & $2.23 \pm 1.77$ & $3.20 \pm 1.40^{*}$ & $3.13 \pm 1.50^{*}$ & $2.25 \pm 1.01$ & $2.21 \pm 1.05$ \\
\hline & $\mathrm{Gl}$ & $1.73 \pm 1.09$ & $2.64 \pm 1.58^{*}$ & $2.57 \pm 1.28^{*}$ & $1.49 \pm 0.61$ & $1.88 \pm 1.30$ \\
\hline & GS & $1.19 \pm 0.52$ & $2.19 \pm 1.22^{*}$ & $2.17 \pm 0.90^{*}$ & $1.79 \pm 0.94$ & $1.50 \pm 0.86$ \\
\hline \multirow[t]{3}{*}{ FENa (\%) } & $\mathrm{GH}$ & $4.00 \pm 3.80$ & $6.58 \pm 7.22^{*}$ & $5.10 \pm 3.48$ & $3.76 \pm 1.90$ & $3.38 \pm 1.66$ \\
\hline & $\mathrm{Gl}$ & $2.99 \pm 3.43$ & $5.37 \pm 6.01^{*}$ & $5.50 \pm 5.99^{*}$ & $3.18 \pm 2.34$ & $2.55 \pm 1.77$ \\
\hline & GS & $1.76 \pm 1.19$ & $2.57 \pm 1.09$ & $2.88 \pm 1.84$ & $2.08 \pm 0.93$ & $4.00 \pm 4.31$ \\
\hline
\end{tabular}

\# $\mathrm{p}<0.05$ : significant difference among groups as compared to $\mathrm{GH}$

${ }^{*} \mathrm{p}<0.05$ : significant difference as compared to control moment of the same group

Table IV - Sodium Para-Aminophurate Clearance (CPAH), Creatinine Clearance (CrC), Filtration Fraction (FF), Plasma Sodium (PNa), Plasma Osmolality (Posm) in Moments of Sudied Groups (Mean \pm SD)

\begin{tabular}{|c|c|c|c|c|c|c|}
\hline \multirow[b]{2}{*}{ Attributes } & \multirow[b]{2}{*}{ Groups } & \multicolumn{5}{|c|}{ Moments } \\
\hline & & Control & Ao15 & Ao30 & DAo15 & DAo30 \\
\hline \multirow[t]{3}{*}{$\mathrm{CPAH}\left(\mathrm{ml} \cdot \mathrm{min}^{-1} \cdot \mathrm{kg}^{-1}\right)$} & $\mathrm{GH}$ & $13.6 \pm 9.4$ & $11.7 \pm 7.1$ & $10.6 \pm 5.4$ & $8.2 \pm 3.4$ & $10.3 \pm 9.0$ \\
\hline & $\mathrm{GI}$ & $15.4 \pm 15.6$ & $15.7 \pm 18.8$ & $10.6 \pm 7.8$ & $11.2 \pm 12.7$ & $15.6 \pm 19.9$ \\
\hline & GS & $14.6 \pm 8.6$ & $15.9 \pm 16.9$ & $14.3 \pm 16.6$ & $13.0 \pm 13.8$ & $8.4 \pm 5.9^{*}$ \\
\hline \multirow[t]{2}{*}{$\mathrm{CrC}\left(\mathrm{ml} \cdot \mathrm{min}^{-1} \cdot \mathrm{kg}^{-1}\right)$} & $\mathrm{GH}$ & $3.6 \pm 1.2$ & $4.0 \pm 2.7$ & $3.9 \pm 2.4$ & $3.9 \pm 2.4$ & $4.0 \pm 2.0$ \\
\hline & GS & $4.1 \pm 1.6$ & $4.5 \pm 2.3$ & $5.5 \pm 4.4$ & $5.2 \pm 3.5$ & $3.5 \pm 2.3$ \\
\hline \multirow[t]{3}{*}{$\mathrm{FF}$} & $\mathrm{GH}$ & $0.35 \pm 0.16$ & $0.39 \pm 0.14$ & $0.38 \pm 0.11$ & $0.48 \pm 0.20$ & $0.47 \pm 0.17$ \\
\hline & $\mathrm{GI}$ & $0.38 \pm 0.21$ & $0.38 \pm 0.15$ & $0.45 \pm 0.19$ & $0.45 \pm 0.29$ & $0.44 \pm 0.24$ \\
\hline & GS & $0.37 \pm 0.19$ & $0.54 \pm 0.48$ & $0.63 \pm 0.44^{*}$ & $0.62 \pm 0.36^{*}$ & $0.63 \pm 0.44^{*}$ \\
\hline \multirow[t]{2}{*}{$\mathrm{PNa}\left(\mathrm{mEq} \cdot \mathrm{L}^{-1}\right)$} & $\mathrm{GH}$ & $139 \pm 2$ & $139 \pm 2$ & $139 \pm 3$ & $137 \pm 2$ & $137 \pm 2$ \\
\hline & GS & $141 \pm 2$ & $140 \pm 2$ & $139 \pm 3$ & $139 \pm 3$ & $138 \pm 2$ \\
\hline \multirow[t]{3}{*}{ Posm (mOsm. kg ${ }^{-1} \mathrm{H}_{2} \mathrm{O}$ ) } & $\mathrm{GH}$ & $291 \pm 24$ & $285 \pm 58$ & $287 \pm 26$ & $283 \pm 33$ & $283 \pm 25$ \\
\hline & $\mathrm{GI}$ & $293 \pm 26$ & $295 \pm 15$ & $287 \pm 35$ & $295 \pm 16$ & $299 \pm 14$ \\
\hline & GS & $289 \pm 18$ & $294 \pm 12$ & $287 \pm 21$ & $285 \pm 19$ & $281 \pm 26$ \\
\hline
\end{tabular}

${ }^{*} p<0.05$ : as compared to control moment in the same group 
Table V - Arterial Arterial ( $\mathrm{pHa})$, Partial Arterial $\mathrm{CO}_{2}$ Pressure $\left(\mathrm{PaCO}_{2}\right)$, Hematocrit $(\mathrm{Ht})$ and Esophageal Temperature $\left(T_{\text {Esoph }}\right)$ within Moments of Studied Groups (Mean $\pm S D$ )

\begin{tabular}{|c|c|c|c|c|c|c|}
\hline \multirow[b]{2}{*}{ Attributes } & \multirow[b]{2}{*}{ Groups } & \multicolumn{5}{|c|}{ Momentos } \\
\hline & & Control & Ao15 & Ao30 & DAo15 & DAo30 \\
\hline \multirow[t]{3}{*}{$\mathrm{pHa}$} & $\mathrm{GH}$ & $7.34 \pm 0.06$ & $7.33 \pm 0.06$ & $7.31 \pm 0.05^{*}$ & $7.31 \pm 0.06^{*}$ & $7.29 \pm 0.06^{*}$ \\
\hline & $\mathrm{GI}$ & $7.35 \pm 0.05$ & $7.33 \pm 0.05^{\star}$ & $7.33 \pm 0.05^{*}$ & $7.31 \pm 0.04^{*}$ & $7.32 \pm 0.05^{\star}$ \\
\hline & GS & $7.32 \pm 0.07$ & $7.31 \pm 0.06$ & $7.31 \pm 0.06$ & $7.28 \pm 0.06^{*}$ & $7.28 \pm 0.05^{\star}$ \\
\hline \multirow[t]{3}{*}{$\mathrm{PaCO}_{2}(\mathrm{mmHg})$} & $\mathrm{GH}$ & $30 \pm 4$ & $30 \pm 6$ & $32 \pm 6$ & $32 \pm 6$ & $33 \pm 6$ \\
\hline & GI & $30 \pm 3$ & $32 \pm 5$ & $30 \pm 6$ & $33 \pm 5$ & $32 \pm 3$ \\
\hline & GS & $34 \pm 4$ & $35 \pm 5$ & $35 \pm 4$ & $35 \pm 4$ & $36 \pm 4$ \\
\hline \multirow[t]{3}{*}{$\mathrm{Ht}(\%)$} & $\mathrm{GH}$ & $37 \pm 8$ & $37 \pm 8$ & $38 \pm 8$ & $38 \pm 8$ & $37 \pm 8$ \\
\hline & $\mathrm{GI}$ & $34 \pm 7$ & $33 \pm 8$ & $33 \pm 8$ & $33 \pm 8$ & $34 \pm 7$ \\
\hline & GS & $32 \pm 6$ & $31 \pm 6$ & $31 \pm 6$ & $32 \pm 7$ & $31 \pm 7$ \\
\hline \multirow[t]{3}{*}{$\mathrm{T}_{\text {Esoph }}(\%)$} & $\mathrm{GH}$ & $36.8 \pm 0.8$ & $36.7 \pm 0.8$ & $36.7 \pm 0.8^{*}$ & $36.6 \pm 0.9^{*}$ & $36.4 \pm 0.8^{*}$ \\
\hline & GI & $37.1 \pm 0.8$ & $36.9 \pm 0.8$ & $36.8 \pm 0.9^{*}$ & $36.7 \pm 0.9^{*}$ & $36.6 \pm 0.9^{*}$ \\
\hline & GS & $37.1 \pm 0.7$ & $36.8 \pm 1.0$ & $36.8 \pm 0.6^{*}$ & $36.7 \pm 0.6^{*}$ & $36.5 \pm 0.7^{*}$ \\
\hline
\end{tabular}

${ }^{*} p<0.05$ as compared to control moment of the same group

\section{DISCUSSION}

This study performed in supposedly healthy animals with volume replacement, has confirmed that aortic crossclamping determines hemodynamic changes, even when performed in distal aorta, such as infra-renal cross-clamping. These changes were characterized in all studied groups by blood pressure and filling pressure increase, such as right atrium and systolic index. So, it seems to have been blood volume redistribution from distal vessels to vessels proximal to aortic occlusion with increase in preload.

Several authors have observed increased filling pressures during aortic cross-clamping ${ }^{27-29}$, while others have not observed such changes ${ }^{30}$. On the other hand, several authors consider the sudden aortic flow impedance increase with after load increase, often followed by cardiac index decrease ${ }^{27}$, the major cause for mean blood pressure increase during aortic cross-clamping ${ }^{31}$. In our study, systemic vascular resistance index was not significantly changed in all groups during aortic cross-clamping.

Quintin etal. ${ }^{29}$ have observed in men, passive venous retreat distally to aortic cross-clamping with catecholamines (epinephrine and norepinephrine) and angiotensin release, determining vasoconstriction both proximal and distal to cross-clamping with capacitance system decrease and blood flow displacement proximal to cross-clamping, with major interference with venous return, that is, preload, depending on aortic cross-clamping being supra or infra-celiac. In the first situation, venous return has always increased because blood flow displacement was toward muscles proximal to cross-clamping, to lungs and brain. In the second situation, there has been blood flow displacement toward splanchnic organs or to other tissues proximal to cross-clamping. If ve- nous splanchnic tone is decreased, there is decreased venous return, but if it is increased, there will be increased venous return. As a consequence, blood distribution between splanchnic and non-splanchnic vessels will determine preload changes.

Renal aortic cross-clamping repercussions were characterized by increased diuresis and natriuresis and decreased urinary osmolarity with all halogenate agents, while with sevoflurane there has been increased renal vascular resistance, but without significant glomerular filtration rate or $\mathrm{CPAAH}$ change, however with filtration fraction increase.

Literature is conflicting about infra-renal aortic crossclamping repercussions on renal function, both in experimental ${ }^{32,33}$ and human ${ }^{4,5}$ studies. Most common results, however, have been increased renal vascular resistance ${ }^{30,32}$ and decreased renal blood flow ${ }^{4,5}$.

Two factors might have been involved in renal function changes found in this experiment: the first is aortic cross-clamping action and the second is anesthetic agent action. Infra-renal aorta occlusion may be associated to renal vascular resistance increase and mild renal blood flow decrease. These hemodynamic changes may persist after unclamping ${ }^{34}$ by decreased glomerular filtration and urinary output. There is a trend for the most external cortex blood flow to further decrease in the most internal cortex, causing blood flow redistribution inside kidneys after occlusive aortic cross-clamping ${ }^{3,6}$. The poor blood flow distribution in the kidneys may persist for up to 60 minutes after infra-renal aortic unclamping ${ }^{4}$.

Colson et al. ${ }^{8}$ have studied the renal effects of anesthesia with isoflurane and halothane in patients submitted to infra-renal aortic cross-clamping. During pre-clamping, renal plasma flow and glomerular filtration were significantly lower 
in patients receiving halothane as compared to those receiving isoflurane. During cross-clamping attributes were not significantly changed in both groups, but glomerular filtration has remained higher in patients under isoflurane. After unclamping, renal attributes were normalized even in the halothane group. This study has shown that anesthesia with halothane is associated to major renal changes before and during aortic cross-clamping suggesting, according to the authors, major renal vasoconstriction. With isoflurane, on the other hand, there has been renal hemodynamic improvement. Since hemodynamic attributes studied before, during and after aortic cross-clamping have not differed between both anesthetic agents, the authors have concluded that benefits observed on renal function of patients receiving isoflurane could not be attributed to isoflurane effects on systemic hemodynamics, but rather to its renal effects.

In a different study with patients submitted to infra-renal aortic cross-clamping under isoflurane, halothane, or droperidol and flunitrazepam, Colson et al. ${ }^{12}$ have observed that isoflurane has prevented aortic cross-clamping renal changes, that is, glomerular filtration rate and urinary volume decrease, which was not true with halothane, flunitrazepam and droperidol. Renal function worsening already in the pre-clamping period in the halothane group suggests major renal vasoconstriction, which might have been mediated by sympathetic nervous system. Isoflurane vasodilation and rennin activity inhibition actions might have also contributed for these results.

Whenever there is extracellular volume expansion with saline solution, such as in our experiment, there will be increased diuresis and decreased urinary osmolality. This happens because kidneys, aiming at incrementing exceeding volume excretion, trigger a complex multifactorial system involving different neuro-humoral pathways ${ }^{35}$. So, the association of volume expansion and preload increase during infra-renal aortic cross-clamping may have caused atrial distension. In response to wall stretching, atrial miocytes increase natriuretic atrial factor secretion which, in addition to causing systemic vasodilation, acts in specific kidney receptors, increases renal sodium and water excretion by inhibiting sodium tubular reabsorption, and increases glomerular filtration rate ${ }^{36}$. Natriuretic factor may also decrease blood pressure by relaxing muscle smooth muscles, and sympathetic stimulation, and inhibit renin-angiotensin-aldosterone system and anti-diuretic hormone release.

Adding to this, there are atrial neural receptors which respond to stretching and transmural pressure changes. There are two mechanoreceptors: type A, located close to large veins entry point and which are not influenced by atrial volume changes; and type B, activated by atrial filling. Stretching and stress signals are detected and conducted by IX and X cranial nerves to medullary and hypothalamic centers, determining integrated physiological responses, among them anti-diuretic hormone release inhibition ${ }^{37}$.

Anti-diuretic hormone, primarily acting on distal and collecting tubules by increasing patency to water, is released with there is osmolarity and external medium volume changes.
Another stimulation for anti-diuretic hormone production is the action of angiotensin II, formed as from renin secretion in areas around the $3^{\text {rd }}$ ventricle. Angiotensin II, in addition to being a potent vasodilator with direct action on vessels smooth muscles, acts on the adrenal gland increasing aldosterone production, the primary action of it is to increase sodium and water reabsorption in the collecting cortical segment.

Most authors have observed increased renin-angiotensin-aldosterone system activity during aortic cross-clamping in dogs ${ }^{38}$ and humans ${ }^{39-41}$. Renin activity increase may be easily explained during supra-renal aortic cross-clamping when there is decreased perfusion pressure of renal afferent arterioles. However, increased rennin activity at infra-renal aortic cross-clamping is not clearly explained by the literature and may be a consequence of the aortic cross-clamping itself, of the anesthetic technique or of volume changes ${ }^{9}$. In our study, the possibility of hormonal activity increase as a consequence of aortic cross-clamping, especially of renin-angiotensin-aldosterone system and anti-diuretic hormone, his impaired due to cardiovascular response and renal changes found, with urinary volume and sodium urinary and fractional excretion increase, without renal vascular resistance increase in the halothane and isoflurane groups. On the other hand, it cannot be totally ruled out in the sevoflurane group, especially renin-angiotensin-aldosterone system activation due to increased renal vascular resistance and filtration fraction, and the absence of significant effect on sodium fractional excretion.

Ma et al. ${ }^{42}$ have observed that sevoflurane has determined in canine renal nerves a more severe dose-dependent depression of slow-conduction C fiber-mediated somatosympathetic reflexes as compared to fast conduction delta A fibers, and only depresses renal spontaneous sympathetic activity in concentrations above $3 \%$. These actions seem to be similar to those determined by isoflurane, but different of those determined by halothane, which depresses in the same proportion somatosympathetic reflexes mediated both by $\mathrm{C}$ and delta A fibers with negligible effects on spontaneous parasympathetic activity, and also different from actions determined by desflurane which in low concentrations is excitatory and in high concentrations equally inhibits reflexes mediated both by $\mathrm{C}$ and delta $\mathrm{A}$ fibers and renal spontaneous sympathetic activity ${ }^{43}$.

This differentiated halogenate anesthetics action on renal nerves spontaneous and sympathetic reflex activity after a certain stimulation, in general algogenic, may help understanding results obtained in renal vasoconstriction with sevoflurane during and after infra-renal aortic cross-clamping.

In theory, halogenate anesthetics promoting vasodilation, such as isoflurane and sevoflurane, would be advantageous in aortic reconstruction surgeries preventing systemic and renal vascular resistance increase during aortic cross-clamping. So, there is virtually a consensus on the indication of isoflurane for vascular surgeries. However, for sevoflurane there is still no well-defined situation for aortic re- 
construction surgeries. According to our results, with renal vascular resistance maintenance in higher levels than baseline during and after cross-clamping and decreased renal plasma and blood flow after unclamping, its indication for this type of vascular surgery seems to be more limited as compared to isoflurane and halothane. Further studies are however needed to check its indication for aortic reconstruction surgeries.

Halothane results were surprising since authors have found major renal changes with this halogenate, be it in the control phase or during aortic cross-clamping. The use of low halogenate concentrations in this study might have influenced results.

Although not using specific markers for renal tubular changes in this study, there are no evidences that there have been renal changes during the control moment, because glomerular and tubular function tests used are within normal parameters for dogs. In addition, sevoflurane concentration limitation in 0.75 MAC and the use of medium fresh gases flows for a limited period of 3 hours were precautions to prevent high fluoride ions and compound A levels. In limiting halogenate concentrations to $0.75 \mathrm{MAC}$, even more important hemodynamic and renal changes, which are probably dose-dependent, were also prevented.

Although care was taken with room temperature, which remained between 24 and $25^{\circ} \mathrm{C}$, and covering dogs with surgical drapes, there has been esophageal temperature decrease in all groups, determining mild to moderate hypothermia because central temperature of the species is 38 to $39^{\circ} \mathrm{C}$

44 . Certainly the use of chilled $5 \%$ glucose solution as cardiac output indicator may also have cooperated for the development of hypothermia, because each result consisted of three consecutive measurements, thus using $30 \mathrm{ml}$ of the indicator in each studied moment, in a total of $150 \mathrm{ml}$ chilled glucose solution infusion during the experiment. Although present, hypothermia intensity was not sufficient to change our results. There has been metabolic acidosis during and after aortic cross-clamping in all groups, probably due to tissue ischemia below cross-clamping.

In conclusion; in dogs and under our experimental conditions, halothane and isoflurane, but not sevoflurane, have minimized major infra-renal aortic cross-clamping complication, which is renal vascular resistance increase.

\section{REFERÊNCIAS - REFERENCES}

01. McCombs PR, Roberts B - Acute renal failure following resection of abdominal aortic aneurysm. Surg Gynecol Obstet, 1979;148:175-178

02. Aronson S, Blumenthal R - Perioperative renal dysfunction and cardiovascular anesthesia: concerns and controversies. J Cardiothor Vasc Anesth, 1998;12:567-586.

03. Youngberg JA - Anesthetic Considerations for Major Vascular Surgery. Annual Refresher Course Lectures. San Francisco, ASA, 1994;511.

04. Gamulin Z, Forster A, Morel D et al - Effects of infrarenal aortic cross-clamping on renal haemodynamics in humans. Anesthesiology, 1984;61:394-399.
05. Awad RW, Barham WJ, Taylor DN et al - The effect of infra-renal aortic reconstruction on glomerular filtration rate and effective renal plasma flow. Eur J Vasc Surg, 1992;6:362-367.

06. Abbot WM, Austen WG - The reversal of renal cortical ischemia during aortic occlusion by mannitol. J Surg Res, 1974;16: 482-489.

07. Paul MD, Mazer CD, Byrick RJ et al - Influence of mannitol and dopamine on renal function during elective infrarenal aortic clamping in man. Am J Nephrol, 1986;6:427-434.

08. Colson P, Capdevilla X, Balert $\mathrm{H}$ et al - Effects of halothane and isoflurane on transient renal dysfunction associated with infrarenal aortic cross-clamping. J Cardiothor Vasc Anesth, 1992;6:295-298.

09. Gelman $S$ - The pathophysiology of aortic cross-clamping and unclamping. Anesthesiology, 1995;82:1026-1060.

10. Alpert RA, Roizen MF, Hamilton WK et al - Intraoperative urinary output does not predict postoperative renal function in patients undergoing abdominal aortic revascularization. Surgery, 1984;95:707-711.

11. Colson P, Capdevilla X, Cuchet D et al - Does choice of the anesthetic influence renal function during infrarenal aortic surgery? Anesth Analg, 1992;74:481-485.

12. Colson P, Ribsteien J, Séguin JR et al - Mechanism of renal hemodynamic impairment during infrarenal aortic cross-clamping. Anesth Analg, 1992;75:18-23.

13. Higuchi $\mathrm{H}$, Arimura $\mathrm{S}$, Sumiura $\mathrm{H}$ et al - Urine concentrating ability after prolonged sevoflurane anesthesia. $\mathrm{Br} \mathrm{J}$ Anaesth, 1994; 73:239-240

14. Mazze RI, Callan CM, Galvez ST et al - The effects of sevoflurane on serum creatinine and blood urea nitrogen concentrations: A retrospective, twenty-two-center, comparative evaluation of renal function in adult surgical patients. Anesth Analg, 2000;90:683-688.

15. Cook TL, Beppu WJ, Hitt BA et al - A comparison of renal effects and metabolism of sevoflurane and methoxyflurane in enzyme induced rats. Anesth Analg, 1975;54:829-834.

16. Malan TP, Kadota $\mathrm{Y}$, Mota $\mathrm{H}$ et al - Renal function after sevoflurane or enflurane anesthesia in the Fischer 344 rat. Anesth Analg, 1993;77:817-821.

17. Jin L, Baillie TA, Davis MR et al - Nephrotoxicity of sevoflurane compound A [fluoromethyl-2,2-difluoro-1 (trifluoro methyl) vinyl ether] in rats: evidence for glutathione and cysteine conjugate formation and the role of renal cysteine conjugate $\beta$-lipase. Biochemical Biophysical Research Communications, 1995;210:498-506.

18. Kharasch ED, Hoffman GM, Thorning D et al - Role of renal cysteine conjugate $\beta$-lyase pathway in inhaled compound $A$ nephrotoxicity in rats. Anesthesiology, 1998;88:1624-1633.

19. Frink EJ, Isner RJ, Malan TP et al - Sevoflurane degradation product concentrations with soda lime during prolonged anesthesia. J Clin Anesth, 1994;74:241-245.

20. Eger II El, Kobliun DD, Bowland T et al - Nephrotoxicity of sevoflurane versus desflurane anesthesia in volunteers. Anesth Analg, 1997;84:160-168.

21. Higuchi H, Sumita $\mathrm{S}$, Wada $\mathrm{H}$ et al - Effects of sevoflurane and isoflurane on renal function and on possible markers of nephrotoxicity. Anesthesiology, 1998;89:307-322.

22. Groudine SB, Fragen RJ, Kharasch ED et al - Comparison of renal function following anesthesia with low-flow sevoflurane and isoflurane. J Clin Anesth, 1999;11:201-207.

23. Cozen FC, Kharasch ED, Czerner FA et al - Low-flow sevoflurane compared with low-flow isoflurane anesthesia in patients with stable renal insufficiency. Anesthesiology, 2002;97:578-584. 
24. Higuchi $\mathrm{H}$, Adachi $\mathrm{Y}$, Wada $\mathrm{H}$ et al - The effects of low-flow sevoflurane and isoflurane anesthesia on renal function in patients with stable moderate renal insufficiency. Anesth Analg, 2001;92:650-655.

25. Kazama T, Ikeda K - Comparison of MAC and the rate of rise of alveolar concentration of sevoflurane with halothane and isoflurane in the dog. Anesthesiology, 1988;68:435-437.

26. Morrison DF - Multivariate Statistical Methods. New York, Mc Graw Hill, 1967;338.

27. Roisen MF, Beaupre PN, Alpert RA et al - Monitoring with two-dimensional transesophageal echocardiography: Comparison of myocardial function in patients undergoing supra-celiac, suprarenal-infraceliac, or infra-renal aortic occlusion. J Vasc Surg, 1984;1:300-305.

28. Harpole DH, Clements FM, Quill T et al - Right and left ventricular performance during and after abdominal aortic aneurysm repair. Ann Surg, 1989;209:356-362.

29. Quintin L, Bonnet F, Macquin I et al - Aortic surgery: effect of clonidine on intra-operative catecholaminergic and circulatory stability. Acta Anaesthesiol Scand, 1990;34:132-137.

30. Seeman-Lodding H, Biber B, Martner J et al - Cardiovascular responses to experimental infra-renal aortic cross-clamping. Modulating effects of isoflurane, sodium nitroprussiate and milrinone. Acta Anaesthesiol Scand, 1996;40:408-415.

31. Kien ND, White DA, Reitan JA et al - The influence of adenosine triphosphate on left ventricular function and blood flow distribution during aortic cross-clamping in dogs. J Cardiothor Vasc Anesth, 1987;1:114-122.

32. Sato JK - Estudo das repercussões hemodinâmicas e renais do pinçamento aórtico infra-renal no cão. Efeitos do pré-tratamento com isradipina. Botucatu, Tese de Doutoramento em Anestesiologia. Faculdade de Medicina de Botucatu, UNESP, 1999;110.

33. Cronenwett JL, Lindenauer SM - Distribution of intrarenal blood flow following aortic clamping and declamping. J Surg Res, 1977;22:469-482.

34. Roberts AJ, Nora JD, Hughes WA et al - Cardiac and renal responses to cross-clamping of the descending thoracic aorta. $J$ Thorac Cardiovasc Surg, 1983;86:732-741.

35. Malnic G - Curso Prático, em: Malnic G, Marcondes M Fisiologia Renal. $3^{a}$ Ed, São Paulo: Ed. Pedagógica e Universitária, 1986;369-390.

36. Rahman SN, Batt AT, Dubose TD et al - Differentiating clinical effects of ANP in oliguric and non-oliguric ATN. J Am Soc Nephrol, 1995;6:474A.

37. Moe GM, Legault L, Skorechi KL - Control of Extracelular Fluid Volume and Pathophysiology of Edema Formation, em: Brenner BM, Rector FC - The Kidney, $4^{\text {th }}$ Ed, Philadelphia, W.B. Saunders, 1991;623-676.

38. Berkowitz HD, Shetty S - Renin release and renal cortical ischemia following aortic cross-clamping. Arch Surg, 1974;109: 612-616.

39. Grindlinger GA, Vegas AM, William GH et al - Independence of renin production and hypertension in abdominal aortic aneurysmectomy. Am J Surg, 1981;141:472-477.

40. Hong SAH, Gelman S, Henderson T - Angiotensin and adrenoceptors in the hemodynamic response to aortic crossclamping. Arch Surg, 1992;127:438-441.
41. Gelman S, Curtis SE, Bradley WE et al - Angiotensin and adrenoreceptors role in hemodynamic response to aortic cross-clamping. Am J Physiol, 1993;264:H14-H20.

42. Ma D, Wang C, Pac-Soo CK et al - The effect of sevoflurane on spontaneous sympathetic activity, $\mathrm{A} \delta$ and $\mathrm{C}$ somatosympathetic reflexes, and associated hemodynamic changes in dogs. Anesth Analg, 1998;86:1079-1083.

43. Pac-Soo CK, Ma D, Wang C et al - Specific actions of halothane, isoflurane, and desflurane on sympathetic activity and $A \delta$ and $C$ somatosympathetic reflexes recorded in renal nerves in dogs. Anesthesiology, 1999;91:470-478.

44. Massone F - Anestesiologia Veterinária. $3^{\mathrm{a}}$ Ed, Rio de Janeiro, Guanabara Koogan, 1999;225.

\section{RESUMEN}

Bisinotto FMB, Braz JRC - Efectos del Halotano, Isoflurano y Sevoflurano sobre la Función Renal en Canes bajo Pinzamiento Aórtico Infra-Renal

JUSTIFICATIVA Y OBJETIVOS: El pinzamiento infra-renal de la aorta abdominal puede producir alteraciones renales. La finalidad del estudio fue evaluar los efectos del halotano, isoflurano y sevoflurano sobre la función renal, en canes sometidos a pinzamiento aórtico infra-renal.

MÉTODO: El estudio aleatorio fue realizado en 30 canes, distribuidos en tres grupos, de acuerdo con el anestésico halogenado utilizado durante la anestesia, en concentraciones equipotentes de 0,75 CAM: $\mathrm{GH}(n=10)$ - halotano a 0,67\%; GI $(n=10)$ - isoflurano a $0,96 \% ; y$ GS $(n=10)$ - sevoflurano a $1,8 \%$. En todos los animales fue realizada ligadura infra-renal de la aorta, por un período de 30 minutos. Los atributos renales fueron estudiados en los momentos: C (control), después 15 (Ao15) y 30 (Ao30) minutos de pinzamiento aórtico, y después 15 (DAo15) y 30 (DAo30) minutos del despinzamiento aórtico.

RESULTADOS: La depuración de agua libre fue menor en los grupos GI y GS, en relación a la GH, después del despinzamiento aórtico $(p<0,05)$. Durante el pinzamiento aórtico, en los tres grupos, hubo aumento del débito urinario, de la excreción urinaria de sodio y de la depuración de sodio, y diminución de la osmolaridad urinaria $(p<0,05)$. La resistencia vascular renal y la fracción de filtración aumentaron solamente en GS $(p<0,05)$, en cuanto la excreción fraccionaria de sodio aumentó en $\mathrm{GH}$ y $\mathrm{Gl}(p<0,05)$. Después del despinzamiento aórtico, hubo normalización de los atributos que se habían alterado, con excepción de la osmolaridad urinaria, que continuó en niveles menores que los del control en todos los grupos $(p<0,05)$. La resistencia vascular renal y la fracción de filtración continuaron más elevadas en GS, acompañadas por diminución del flujo sanguíneo renal y de la depuración de para-aminohipurato de sodio $(p<0,05)$.

CONCLUSIONES: En el can, en las condiciones experimentales empleadas, la inhalación de halotano e isoflurano a 0,75 CAM, más no de sevoflurano, atenuó la principal alteración después del pinzamiento infra-renal de la aorta, que es un aumento de la resistencia vascular renal. 